\title{
Power Operations and Coactions in Highly Commutative Homology Theories
}

\author{
by \\ Andrew BAKER \\ This paper is dedicated to the memory of my friend Goro Nishida (1943-2014), \\ whose pioneering work on power operations inspired it.
}

\begin{abstract}
Power operations in the homology of infinite loop spaces, and $H_{\infty}$ or $E_{\infty}$ ring spectra have a long history in algebraic topology. In the case of ordinary mod $p$ homology for a prime $p$, the power operations of Kudo, Araki, Dyer and Lashof interact with Steenrod operations via the Nishida relations, but for many purposes this leads to complicated calculations once iterated applications of these functions are required. On the other hand, the homology coaction turns out to provide tractable formulae better suited to exploiting multiplicative structure.

We show how to derive suitable formulae for the interaction between power operations and homology coactions in a wide class of examples; our approach makes crucial use of modern frameworks for spectra with well behaved smash products. In the case of mod $p$ homology, our formulae extend those of Bisson and Joyal to odd primes. We also show how to exploit our results in sample calculations, and produce some apparently new formulae for the Dyer-Lashof action on the dual Steenrod algebra.
\end{abstract}

2010 Mathematics Subject Classification: Primary 55S12; Secondary 55P42, 55S10.

Keywords: power operations, coactions, $E_{\infty}$ and $H_{\infty}$ ring spectra.

\section{Introduction}

In this note we study the interaction between coactions over homology Hopf algebroids (such as the Steenrod algebra for a prime $p$ ) and power operations (such as Dyer-Lashof operations). Some of our results are surely known, but we are only aware of partial references such as $[4,5]$ which only deal with the case of ordinary

Communicated by T. Ohtsuki. Received August 14, 2014. Revised November 17, 2014, December 16, 2014, and December 18, 2014.

A. Baker: School of Mathematics \& Statistics, University of Glasgow, Glasgow G12 8QW, Scotland; http://www.maths.gla.ac.uk/ ajb; e-mail: a.baker@maths.gla.ac.uk 
mod 2 homology. In any case, our approach to understanding this relationship involves a modern perspective based on a symmetric monoidal category of spectra with good properties such as that of [10].

The examples we discuss in detail are based on ordinary $\bmod p$ homology for a prime $p$ and the power operations originally introduced by Kudo, Araki, Dyer and Lashof, then generalised by May et al. [9, 12, 7], usually rather unhistorically referred to as Dyer-Lashof operations. Studying the interaction between the coaction and the Dyer-Lashof operations amounts to studying dual versions of the classical Nishida relations [17]. We use knowledge of the coaction of the dual Steenrod algebra $\mathcal{A}(p)_{*}$ to investigate the homology of commutative $S$-algebras $R$ where $\pi_{0}(R)$ has characteristic $p$. Of course such questions were studied by Steinberger [7, Chapter III]. However, our approach offers some clarification of the algebra involved in the Dyer-Lashof action on the dual Steenrod algebra itself, relating it to work of Kochman [11] (see also [20]); our detailed knowledge of the homology also allows us to give a refined version of Steinberger's splitting result, giving more information on the multiplicative structure.

The results of this paper have been used in joint work with Rolf Hoyer and some were an outcome of discussions with him.

\section{Notation, etc.}

We will use the floor and ceiling functions $\lfloor-\rfloor,\lceil-\rceil: \mathbb{R} \rightarrow \mathbb{Z}$ taking values

$$
\lfloor x\rfloor=\max \{n \in \mathbb{Z}: n \leqslant x\}, \quad\lceil x\rceil=\min \{n \in \mathbb{Z}: n \geqslant x\} .
$$

In particular, for $x \in \mathbb{Z}$ we have $\lfloor x\rfloor=\lceil x\rceil=x$, while if $x \notin \mathbb{Z}$, then $\lceil x\rceil=\lfloor x\rfloor+1$.

When working with power series $f(t)$ in an indeterminate $t,[f(t)]_{t^{n}}$ will denote the coefficient of $t^{n}$ in $f(t)$.

\section{Bimodules}

We will often consider bimodules. If $\mathrm{R}, \mathrm{R}^{\prime}, \mathrm{R}^{\prime \prime}$ are three rings, $\mathrm{M}$ is an $\mathrm{R}-\mathrm{R}^{\prime}-$ bimodule, and $\mathrm{N}$ is an $\mathrm{R}^{\prime}-\mathrm{R}^{\prime \prime}$-bimodule, then we will denote the tensor product over $R^{\prime}$ by $M \otimes_{R^{\prime}} N$. We will reserve $\otimes_{R}$ for the situation where $R$ is commutative and $\mathrm{U}, \mathrm{V}$ are two left R-modules and denote their tensor product by $\mathrm{U} \otimes_{\mathrm{R}} \mathrm{V}$. We will sometimes consider a left $\mathrm{R}_{*}$-module $\mathrm{M}_{*}$ over a graded commutative ring $\mathrm{R}_{*}$ as having a canonical right $\mathrm{R}_{*}$-module structure given by

$$
m \cdot r=(-1)^{|r| \cdot|m|} r m
$$

for homogeneous elements $r \in \mathrm{R}_{|r|}$ and $m \in \mathrm{M}_{|m|}$. 


\section{Bigebroids and comodules}

Suppose that A, B, H are commutative (graded) rings and that

$$
A \stackrel{\eta_{\mathrm{A}}}{\longrightarrow} \mathrm{H} \stackrel{\eta_{\mathrm{B}}}{\longleftarrow} \mathrm{B}
$$

are ring homomorphisms. We use these to define a left A-module structure and a right $\mathrm{B}$-module structure on $\mathrm{H}$. Given a right $\mathrm{A}$-module $\mathrm{M}$ and a left B-module $\mathrm{N}$, we can define the bimodule tensor products

$$
\mathrm{M} \otimes_{\mathrm{A}} \mathrm{H}, \quad \mathrm{H} \otimes_{\mathrm{B}} \mathrm{N}, \quad \mathrm{M} \otimes_{\mathrm{A}} \mathrm{H} \otimes_{\mathrm{B}} \mathrm{N} .
$$

If $\mathrm{R}$ is a commutative graded ring, then its opposite ring has as its underlying set $\mathrm{R}^{\mathrm{op}}=\mathrm{R}$ and multiplication of homogeneous elements given by

$$
x^{\mathrm{op}} y^{\mathrm{op}}=( \pm)(y x)^{\mathrm{op}},
$$

where the sign is determined in the usual way in terms of the degrees of $x, y$. The opposite ring $\mathrm{H}^{\mathrm{op}}$ admits a right $\mathrm{A}^{\mathrm{op}}$-module structure and a left $\mathrm{B}^{\mathrm{op}}$-module structure and there is a ring isomorphism

$$
\mathrm{R} \stackrel{\cong}{\rightrightarrows} \mathrm{R}^{\mathrm{op}}, \quad x \leftrightarrow( \pm) x^{\mathrm{op}},
$$

which interchanges the two pairs of module structures.

\section{Part I}

\section{Power operations and coactions}

\section{$\S 1$. Extended powers and power operations}

In this section we give some general observations on extended powers. We will work in the category $\mathscr{M}_{S}$ of $S$-modules of [10] and write $\wedge$ for $\wedge_{S}$. For an $S$-module $M$,

$$
M^{\wedge n}=M \wedge \cdots \wedge M \text {. }
$$

For an $S$-module $N$ with a left $\Sigma_{n}$-action we will denote the half-smash product by $E \Sigma_{n} \ltimes_{\Sigma_{n}} N$. In particular we will write

$$
D_{n} M=E \Sigma_{n} \ltimes_{\Sigma_{n}} M^{\wedge n}
$$

for the extended power, and when $G \leqslant \Sigma_{n}$, we will sometimes set

$$
D_{G} M=E \Sigma_{n} \ltimes_{G} M^{\wedge n} .
$$

If $M$ is cofibrant then by [10, Theorem III.5.1], the projection of $E \Sigma_{n}$ to a point induces a weak equivalence

$$
D_{n} M=E \Sigma_{n} \ltimes_{\Sigma_{n}} M^{\wedge n} \stackrel{\sim}{\longrightarrow} M^{\wedge n} / \Sigma_{n} .
$$


More generally, if $R$ is a cofibrant commutative $S$-algebra, then in the category $\mathscr{M}_{R}$ of $R$-modules, for an $R$-module $N$ we can define

$$
D_{n}^{R} N=E \Sigma_{n} \ltimes_{\Sigma_{n}} N^{\wedge_{R} n},
$$

and if $N$ is a cofibrant $R$-module, the natural map gives a weak equivalence

$$
D_{n}^{R} N=E \Sigma_{n} \ltimes_{\Sigma_{n}} N^{\wedge_{R} n} \stackrel{\sim}{\rightarrow} N^{\wedge_{R} n} / \Sigma_{n}
$$

If $M \in \mathscr{M}_{S}$, there is an isomorphism

$$
R \wedge D_{n} M \cong D_{n}^{R}(R \wedge M) .
$$

Now we recall the definition of power operations. We will do this in a general setting, for three commutative $S$-algebras $A, B, E$ (actually, it is enough to assume that $E$ is an $H_{\infty}$ ring spectrum). There is a map $\mu_{n}: D_{n} E \rightarrow E$ which induces a diagram of $A$-module morphisms.

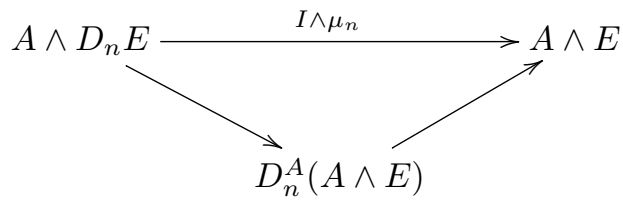

If $x: S^{m} \rightarrow A \wedge E$, then the composition of solid arrows in the commutative diagram

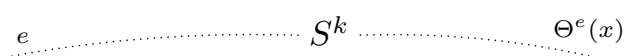

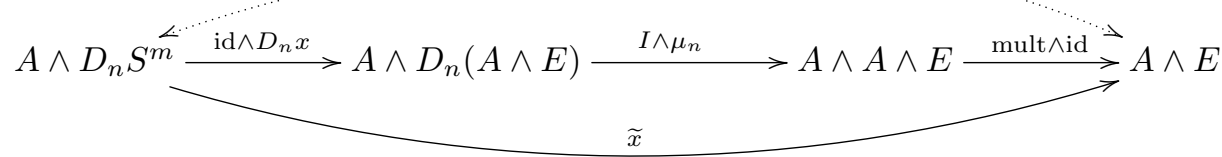

defines a power operation

$$
\Theta^{e}: A_{m}(E) \rightarrow A_{k}(E), \quad \Theta^{e}(x)=\widetilde{x}_{*} e,
$$

for each element $e \in A_{k}\left(D_{n} S^{m}\right)=\pi_{k}\left(A \wedge D_{n} S^{m}\right)$.

\section{$\S 2$. Generalised coactions}

For any $S$-module $X$, we can use the unit $S \rightarrow B$ and switch maps to induce the horizontal morphisms in the following commutative diagram:

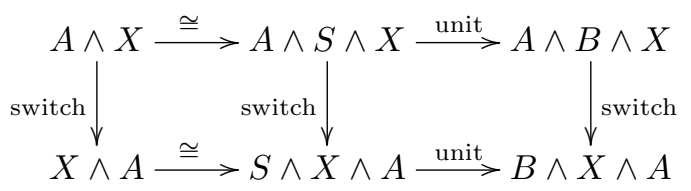


On applying homotopy $\pi_{*}(-)$ we obtain maps similar to coactions, at least when suitable flatness conditions hold.

We make an algebraic assumption: the left $B_{*}$-module $B_{*}(A)=\pi_{*}(B \wedge A)$ is flat. Then on passing to homotopy groups we find that there is an isomorphism of left $B_{*}$-modules

$$
B_{*}(X \wedge A) \stackrel{\cong}{\longrightarrow} B_{*}(X) \otimes_{B_{*}} B_{*}(A),
$$

and an isomorphism

$$
A_{*}(B \wedge X) \stackrel{\cong}{\longrightarrow} A_{*}(B) \bigotimes_{B_{*}} B_{*}(X) .
$$

The rightmost switch map induces an isomorphism

$$
A_{*}(B \wedge X) \stackrel{\cong}{\longrightarrow} B_{*}(X) \otimes_{B_{*}} B_{*}(A)
$$

which converts the left $A_{*}$-module structure to a right module structure. These ingredients give the following commutative diagram:

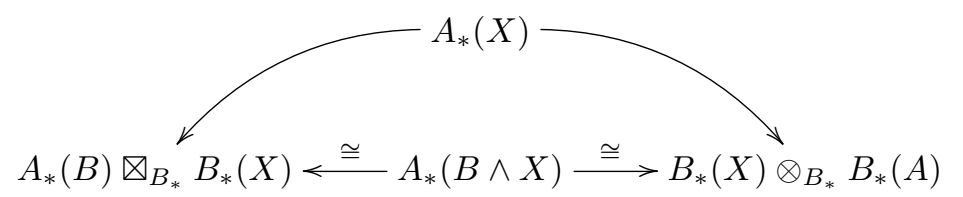

If $A=B$, then in

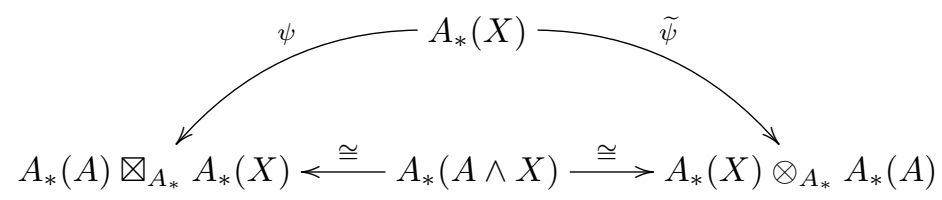

the homomorphism $\psi$ is the usual left $A_{*}(A)$-coaction on $A_{*}(X)$, while $\widetilde{\psi}$ is obtained by composing $\psi$ with the antipode of the Hopf algebroid $A_{*}(A)$ and a switch map. In fact $\widetilde{\psi}$ is a right coaction making $A_{*}(X)$ into a right $A_{*}(A)$-comodule. If we also take $E=A$, then for each $e \in A_{k}\left(D_{n} S^{m}\right)$ there is a power operation $\Theta^{e}$ as in (1.3), but also another obtained by interchanging the rôles of the two factors of $A$,

$$
\widetilde{\Theta}^{e}=\chi \Theta^{e} \chi
$$

where $\chi: A_{*}(A) \rightarrow A_{*}(A)$ is the antipode induced by the switch map on $A \wedge A$.

The unit $S \rightarrow B$ induces the downward morphisms in the following commutative diagram: 


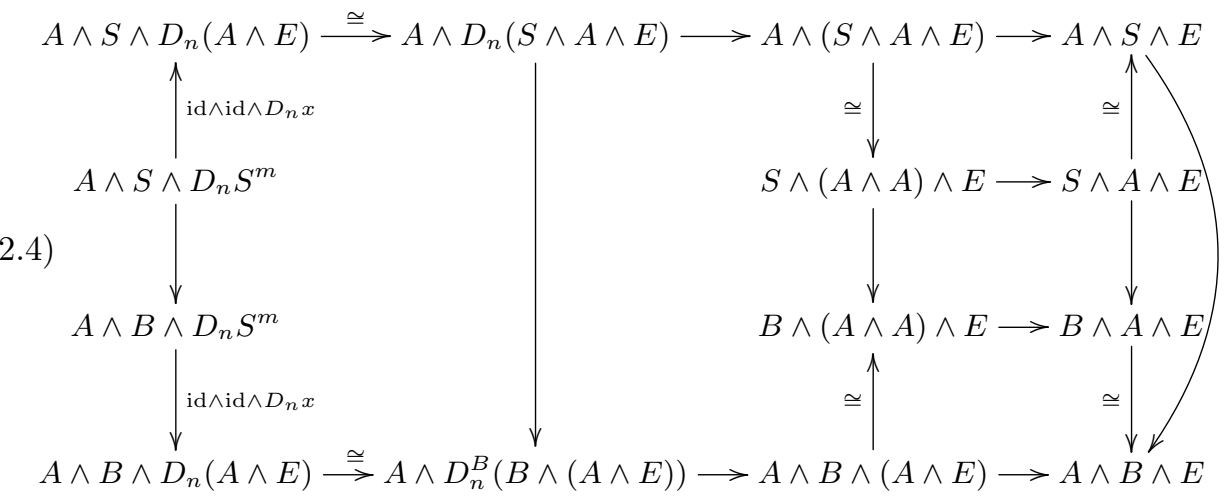

On applying $\pi_{*}(-)$ to this diagram we obtain an algebraic analogue:

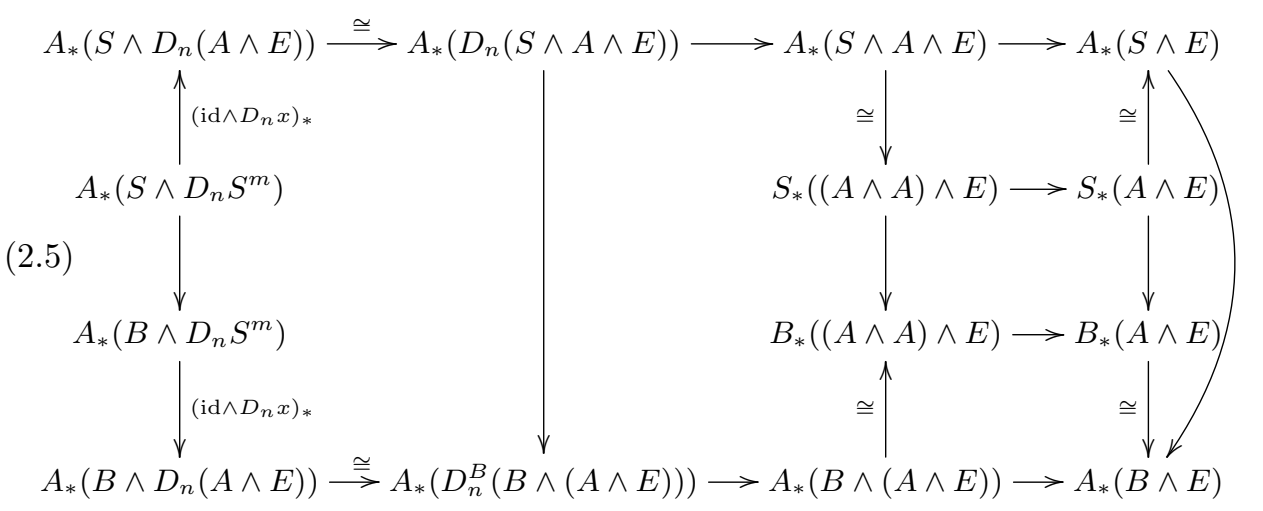

When $B=A$ and $A_{*}(A)$ is $A_{*}$-flat, $\left(A_{*}, A_{*}(A)\right)$ has the structure of a Hopf algebroid. For any spectrum $X$, the unit $S \rightarrow A$ induces a map

$$
A \wedge X \stackrel{\cong}{\rightrightarrows} A \wedge S \wedge X \rightarrow A \wedge A \wedge X
$$

and there is a left coaction

$$
\psi: A_{*}(X) \rightarrow A_{*}(A) \otimes_{A_{*}} A_{*}(X)
$$

which fits into the following commutative diagram:

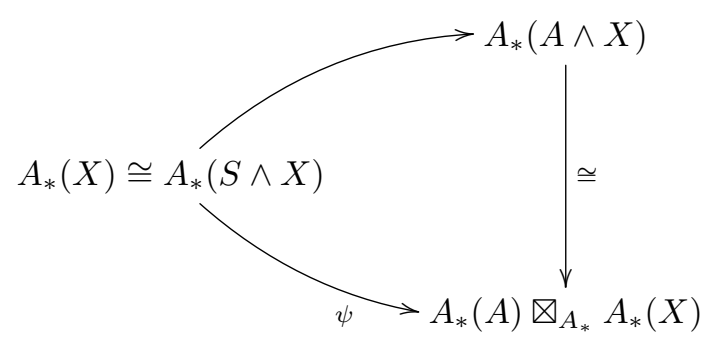


In this situation, (2.5) can be used to study the $A_{*}(A)$-coaction and its relationship with power operations defined above. Taking an element

$$
e \in A_{*}\left(D_{n} S^{m}\right) \cong A_{*}\left(S \wedge D_{n} S^{m}\right)
$$

and chasing it upwards to the right and downwards to

$$
A_{*}(A \wedge E) \cong A_{*}(A) \bigotimes_{A_{*}} A_{*}(E)
$$

and then comparing the result with that obtained by going downwards to the right,

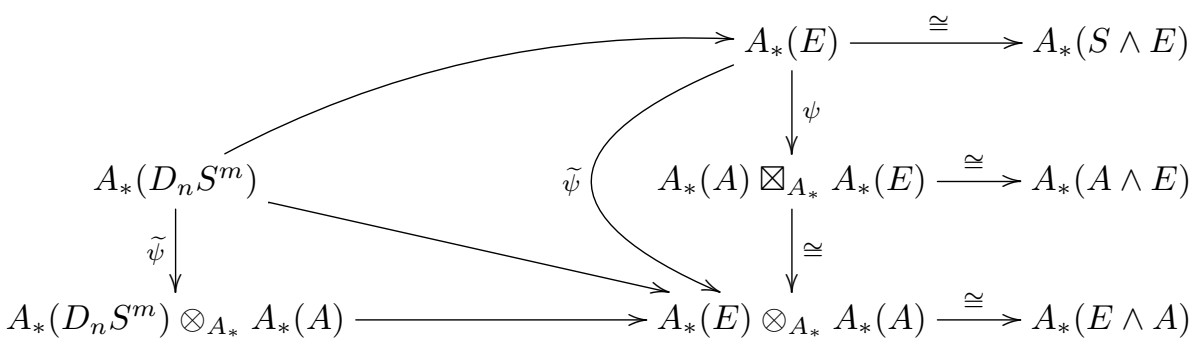

we obtain the following important formula:

$$
\widetilde{\psi}\left(\Theta^{e}(x)\right)=\sum_{i}\left(1 \otimes \chi\left(\theta_{i}\right)\right) \Theta^{e_{i}}(\widetilde{\psi} x)
$$

where $\psi(e)=\sum_{i} \theta_{i} \otimes e_{i}$.

\section{$\S 3$. Further generalisations}

The situation of the previous sections can be generalised somewhat. Suppose that $M$ is a right $A$-module. Then we can replace the element of $A_{k}\left(D_{n} S^{m}\right)$ with $e \in M_{k}\left(D_{n} S^{m}\right)$ and use the composition

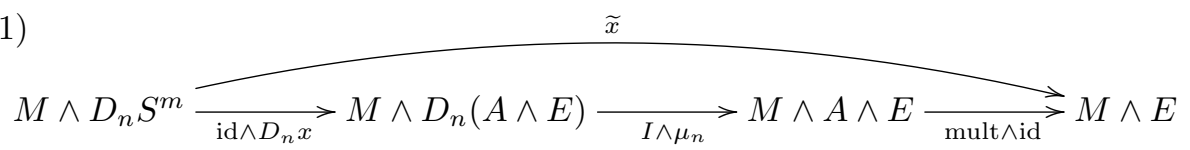

to define a power operation

$$
\Theta^{e}: A_{m}(E) \rightarrow M_{k}(E), \quad \Theta^{e}(x)=\widetilde{x}_{*} e,
$$

analogous to that of (1.3).

In order to get a sensible notion of left coaction $M_{*}(X) \rightarrow M_{*}(B) \bigotimes_{B_{*}} B_{*}(X)$ leading to analogues of the formulae above in good situations, it is necessary to assume that $B_{*}(A)$ is $B_{*}$-flat, and also that one of the following conditions holds:

- $A_{*}(X)$ is $A_{*}$-flat;

- $M_{*}$ is $A_{*}$-flat as a right $A_{*}$-module. 
When $B=A$, the assumptions that $A_{*}(A)$ is flat as a left or right $A_{*}$-module are equivalent, and in the most important cases in algebraic topology this holds for any $M$. We leave the interested reader to work out the details. Such operations are likely to be hard to work with unless $M$ has suitable multiplicative structure (e.g., it is a commutative $A$-algebra).

One important class of examples is that where $A=B=E_{n}$, the $n$-th LubinTate spectrum for a prime $p$, and $M=K_{n}$, the $n$-th Morava $K$-theory. In this case, $K_{n}$ is an $E_{n}$ ring spectrum (non-homotopy commutative if $p=2$ ); more generally, we could take $M=E_{n} \wedge W$, where $W$ is a generalised Moore spectrum as in [8]. The work of the latter suggests defining power operations using prosystems of such operations; this is presumably related to the work of McClure [7, Chapter IX] on power operations in $K$-theory.

\section{Part II}

\section{Eilenberg-Mac Lane spectra and Dyer-Lashof operations}

\section{§4. Eilenberg-Mac Lane spectra and the dual Steenrod algebra}

In this section we discuss the important case of the Eilenberg-Mac Lane spectrum for a prime $p$ and take $A=B=H=H \mathbb{F}_{p}$. The dual Steenrod algebra $\mathcal{A}_{*}=$ $\mathcal{A}(p)_{*}=H_{*}(H)$ is actually a Hopf algebra over $\pi_{*}(H)=\mathbb{F}_{p}$ since the two unit homomorphisms coincide. We will usually write $\otimes=\otimes_{\mathbb{F}_{p}}$ in place of $\bigotimes_{\mathbb{F}_{p}}$ as there is no danger of confusion. The above isomorphism

$$
H_{*}(H) \bigotimes_{\mathbb{F}_{p}} H_{*}(X) \stackrel{\cong}{\longrightarrow} H_{*}(X) \otimes_{\mathbb{F}_{p}} H_{*}(H)
$$

coincides with the composition switch $\circ(\chi \otimes I)$, and

$$
\widetilde{\psi}=\operatorname{switch} \circ(\chi \otimes I) \circ \psi \text {. }
$$

On a basic tensor $\alpha \otimes x \in H_{*}(H) \otimes H_{*}(X)$ this gives

$$
\alpha \otimes x \leftrightarrow(-1)^{|\alpha| \cdot|x|} x \otimes \chi(\alpha) .
$$

The Steenrod algebra $\mathcal{A}^{*}$ is the $\mathbb{F}_{p^{-}}$-linear dual of $\mathcal{A}_{*}$ with associated dual pairing

$$
\langle-\mid-\rangle: \mathcal{A}^{*} \otimes \mathcal{A}_{*} \rightarrow \mathbb{F}_{p}
$$

This gives rise to a right action of $\mathcal{A}^{*}$ on a left $\mathcal{A}_{*}$-comodule $M_{*}$ by

$$
a_{*} x=x \cdot a=(-1)^{|a| \cdot|x|} \sum_{i}\left\langle a \mid \gamma_{i}\right\rangle x_{i},
$$


where $a \in \mathcal{A}^{*}, x \in M_{*}$ and $\psi x=\sum_{i} \gamma_{i} \otimes x_{i}$. There is also a dual pairing

$$
\langle-\mid-\rangle: \mathcal{A}_{*} \otimes \mathcal{A}^{*} \rightarrow \mathbb{F}_{p} \quad \text { defined by } \quad\langle\alpha \mid a\rangle=(-1)^{|\alpha| \cdot|a|}\langle a \mid \chi \alpha\rangle,
$$

giving an alternative formulation of the right action as

$$
a_{*} x=\sum_{i}\left\langle\gamma_{i}^{\prime} \mid a\right\rangle x_{i}, \quad \text { where } \quad \tilde{\psi} x=\sum_{i} x_{i} \otimes \gamma_{i}^{\prime} .
$$

$\S 4.1$. The case $p=2$

When $A=B=H=H \mathbb{F}_{2}$, the dual Steenrod algebra is

$$
\mathcal{A}_{*}=\mathbb{F}_{2}\left[\xi_{r}: r \geqslant 1\right]=\mathbb{F}_{2}\left[\zeta_{r}: r \geqslant 1\right],
$$

where the Milnor generator $\xi_{r} \in \mathcal{A}_{2^{r}-1}$ is defined to be the image of the generator of $H_{2^{r}-1}\left(\mathbb{R} \mathrm{P}^{\infty}\right)$ under the homomorphism induced by the canonical map $\mathbb{R} \mathrm{P}^{\infty} \rightarrow$ $\Sigma H \mathbb{F}_{2}$, and $\zeta_{r}=\chi\left(\xi_{r}\right)$ is its conjugate; by convention $\xi_{0}=\zeta_{0}=1$. We will make use of the generating series

$$
\xi(t)=t+\sum_{r \geqslant 1} \xi_{r} t^{2^{r}}, \quad \zeta(t)=t+\sum_{r \geqslant 1} \zeta_{r} t^{t^{r}}
$$

which are composition inverses, i.e., $\zeta(\xi(t))=t=\xi(\zeta(t))$.

We have

$$
H_{2 m+r}\left(D_{2} S^{m}\right)=\left\{\begin{array}{cl}
\mathbb{F}_{2} & \text { if } r \geqslant 0, \\
0 & \text { otherwise }
\end{array}\right.
$$

and the generator in degree $r+2 m$ gives the operation $\mathrm{Q}_{r}=\mathrm{Q}^{r+m}$. We write $\widetilde{\mathrm{Q}}_{r}=\widetilde{\mathrm{Q}}^{r+m}$ for the twisted version of these as in (2.3), so

$$
\widetilde{\mathrm{Q}}_{r}=\chi \mathrm{Q}_{r} \chi=\chi \mathrm{Q}^{r+m} \chi=\widetilde{\mathrm{Q}}^{r+m} .
$$

Theorem 4.1. Let $x \in H_{m}(E)$ and $\psi(x)=\sum_{i} \alpha_{i} \otimes x_{i}$. Then

$$
\sum_{m \leqslant r} \psi\left(\mathrm{Q}^{r} x\right) t^{r}=\sum_{m \leqslant k} \sum_{0 \leqslant j \leqslant k} \sum_{i} \xi(t)^{k} \widetilde{\mathrm{Q}}^{j} \alpha_{i} \otimes \mathrm{Q}^{k-j} x_{i},
$$

or equivalently

$$
\psi\left(\mathrm{Q}^{r} x\right)=\sum_{m \leqslant k} \sum_{0 \leqslant j \leqslant k} \sum_{i}\left[\xi(t)^{k}\right]_{t^{r}} \widetilde{\mathrm{Q}}^{j} \alpha_{i} \otimes \mathrm{Q}^{k-j} x_{i} .
$$

Proof. We recall that for $m \in \mathbb{Z}$, there is a weak equivalence

$$
D_{2} S^{m} \stackrel{\sim}{\rightarrow} \Sigma^{m} \mathbb{R P}_{m}^{\infty},
$$


where $\mathbb{R P}_{m}^{\infty}$ is the Thom spectrum of the virtual bundle $m \lambda$, and $\lambda \downarrow \mathbb{R P}{ }^{\infty}$ is the canonical real line bundle associated to the real sign representation of $\Sigma_{2}$. When $m \geqslant 0$,

$$
\mathbb{R P}_{m}^{\infty}=\mathbb{R P ^ { \infty } / R P ^ { m - 1 }}
$$

Writing $\bar{e}_{r+m}(r \geqslant 0)$ for the image of the generator $e_{r} \in H_{r}\left(\mathbb{R P}{ }^{\infty}\right)$ under the Thom isomorphism

$$
H_{*}\left(\mathbb{R P}^{\infty}\right) \cong H_{*+m}\left(\mathbb{R P}_{m}^{\infty}\right)
$$

the coaction is given by

$$
\sum_{r \geqslant 0} t^{r+m} \psi \bar{e}_{r+m}=\sum_{s \geqslant 0} \xi(t)^{s+m} \otimes \bar{e}_{s+m} .
$$

Under the composition of the isomorphisms

$$
H_{*}\left(D_{2} S^{m}\right) \stackrel{\cong}{\rightrightarrows} H_{*}\left(\Sigma^{m} \mathbb{R P} P_{m}^{\infty}\right) \cong H_{*-m}\left(\mathbb{R P}_{m}^{\infty}\right)
$$

induced by the above equivalence, the following elements correspond:

$$
e_{r} \otimes x_{m}^{\otimes 2} \leftrightarrow \bar{e}_{r+m}
$$

where $x_{m} \in H_{m}\left(S^{m}\right)$ is the generator. Now the result follows from (2.6), which gives the following in terms of the right coaction $\widetilde{\psi}$ :

$$
\sum_{m \leqslant r} \widetilde{\psi}\left(\mathrm{Q}^{r} x\right) t^{r}=\sum_{m \leqslant k} \mathrm{Q}^{k}(\widetilde{\psi} x)\left(1 \otimes \zeta(t)^{k}\right)
$$

We will sometimes use generating functions to express such formulae. For example, we have the series

$$
\mathrm{Q}_{t}=\sum_{r \in \mathbb{Z}} \mathrm{Q}^{r} t^{r}
$$

and on substituting $\zeta(t)$ for $t$,

$$
\mathrm{Q}_{\zeta(t)}=\sum_{r \in \mathbb{Z}} \mathrm{Q}^{r} \zeta(t)^{r}
$$

Then

$$
\widetilde{\psi} \mathrm{Q}_{t} x=\sum_{|x| \leqslant r} \widetilde{\psi}\left(\mathrm{Q}^{r} x\right) t^{r}=\sum_{|x| \leqslant r} \mathrm{Q}^{r}(\widetilde{\psi} x) \zeta(t)^{r}=\mathrm{Q}_{\zeta(t)}(\widetilde{\psi} x) .
$$

The following formulae for Dyer-Lashof operations at the prime 2 are due to Steinberger [7, Theorem III.2.2]. 
Theorem 4.2. For $r, s \geqslant 1$,

$$
\mathrm{Q}^{2^{s}-2} \zeta_{1}=\zeta_{s}, \quad \mathrm{Q}^{r} \zeta_{1} \neq 0, \quad \mathrm{Q}^{r} \zeta_{s}= \begin{cases}\mathrm{Q}^{r+2^{s}-2} \zeta_{1} & \text { if } r \equiv 0,-1\left(\bmod 2^{s}\right), \\ 0 & \text { otherwise. }\end{cases}
$$

Corollary 4.3. For $s, t \geqslant 1$,

$$
\widetilde{\mathrm{Q}}^{2^{s}-2} \xi_{1}=\xi_{s}, \quad \widetilde{\mathrm{Q}}^{r} \xi_{1} \neq 0, \quad \widetilde{\mathrm{Q}}^{r} \xi_{s}= \begin{cases}\widetilde{\mathrm{Q}}^{r+2^{s}-2} \xi_{1} & \text { if } r \equiv 0,-1\left(\bmod 2^{s}\right), \\ 0 & \text { otherwise. }\end{cases}
$$

For later use we record a result that may be known but we know of no reference.

Lemma 4.4. For $s \geqslant 1$,

$$
\mathrm{Q}^{2^{s}} \xi_{s}=\xi_{s+1}+\xi_{1} \xi_{s}^{2}
$$

Proof. Before proving this we note that if $1 \leqslant r \leqslant s$, then for degree reasons

$$
\mathrm{Q}^{2^{s}}\left(\zeta_{r} \xi_{s-r}^{2^{r}}\right)=\left(\mathrm{Q}^{2^{r}-1} \zeta_{r}\right) \mathrm{Q}^{2^{s}-2^{r}+1}\left(\xi_{s-r}^{2^{r}}\right)+\left(\mathrm{Q}^{2^{r}} \zeta_{r}\right) \mathrm{Q}^{2^{s}-2^{r}}\left(\xi_{s-r}^{2^{r}}\right)=\zeta_{r+1} \xi_{s-r}^{2^{r+1}} .
$$

Suppose that (4.2) is true for $s<n$. By definition of the antipode $\chi$, and using Theorem 4.2 we obtain

$$
\begin{aligned}
\mathrm{Q}^{2^{n}} \xi_{n} & =\mathrm{Q}^{2^{n}}\left(\zeta_{n}+\zeta_{n-1} \xi_{1}^{2^{n-1}}+\cdots+\zeta_{1} \xi_{n-1}^{2}\right) \\
& =\zeta_{n+1}+\left(\mathrm{Q}^{2^{n-1}} \zeta_{n-1}\right) \xi_{1}^{2^{n}}+\cdots+\left(\mathrm{Q}^{2} \zeta_{1}\right) \xi_{n-1}^{2^{2}} \\
& =\zeta_{n+1}+\zeta_{n} \xi_{1}^{2^{n}}+\cdots+\zeta_{2} \xi_{n-1}^{2^{2}} \\
& =\left(\zeta_{n+1}+\zeta_{n} \xi_{1}^{2^{n}}+\cdots+\zeta_{2} \xi_{n-1}^{2^{2}}+\zeta_{1} \xi_{n}^{2}\right)+\zeta_{1} \xi_{n}^{2} \\
& =\xi_{n+1}+\xi_{1} \xi_{n}^{2} .
\end{aligned}
$$

\section{$\S 4.2$. The case of an odd prime}

Suppose that $p$ is an odd prime and $A=H=H \mathbb{F}_{p}$. The dual Steenrod algebra is

$$
\mathcal{A}_{*}=\mathbb{F}_{p}\left[\xi_{r}: r \geqslant 1\right] \otimes \Lambda\left(\tau_{s}: s \geqslant 0\right)=\mathbb{F}_{p}\left[\zeta_{r}: r \geqslant 1\right] \otimes \Lambda\left(\bar{\tau}_{s}: s \geqslant 0\right)
$$

where the Milnor generators $\xi_{r} \in \mathcal{A}_{2\left(p^{r}-1\right)}$ and $\tau_{r} \in \mathcal{A}_{2 p^{r}-1}$ are the images of generators of $H_{2 p^{r}-1}\left(B C_{p}\right)$ and $H_{2 p^{r}}\left(B C_{p}\right)$ under the homomorphism induced by the canonical map $B C_{p} \rightarrow \Sigma H \mathbb{F}_{p}$, and $\zeta_{r}=\chi\left(\xi_{r}\right), \bar{\tau}_{r}=\chi\left(\tau_{r}\right)$ are their conjugates; by convention $\xi_{0}=\zeta_{0}=1$.

For $m \in \mathbb{Z}$

$$
H_{2 m p+2 r(p-1)-\varepsilon}\left(D_{p} S^{2 m}\right)= \begin{cases}\mathbb{F}_{p} & \text { if } r \geqslant 0 \text { and } \varepsilon=0, \\ \mathbb{F}_{p} & \text { if } r \geqslant 1 \text { and } \varepsilon=1, \\ 0 & \text { otherwise }\end{cases}
$$


and a suitably chosen generator in degree $2 m p+2 r(p-1)-\varepsilon$ gives rise to the operation $\beta^{\varepsilon} \mathrm{Q}_{r}=\beta^{\varepsilon} \mathrm{Q}^{r+m}$.

In order to give a similar discussion to that for the case $p=2$, we follow the outline of [7, Section V.2]. Let

$$
W=\left\{\left(x_{1}, \ldots, x_{p}\right) \in \mathbb{R}^{p}: x_{1}+\cdots+x_{p}=0\right\}
$$

be the reduced real permutation representation of $\Sigma_{p}$ in which not all elements act orientably, although $C_{p} \leqslant \Sigma_{p}$ does act by preserving orientations. Given any finite-dimensional real vector space $U$, we can view $U^{p}=U \oplus \cdots \oplus U$ with the permutation action of $\Sigma_{p}$ as equivalent to

$$
(\mathbb{R} \oplus W) \otimes_{\mathbb{R}} U \cong U \oplus\left(W \otimes_{\mathbb{R}} U\right)
$$

with $\Sigma_{p}$ acting only on the left hand factor and second summands respectively. As $C_{p}$-representations,

$$
W \cong W_{1} \oplus \cdots \oplus W_{(p-1) / 2}
$$

where $W_{r}=\mathbb{R}^{2}$ with the generator of $C_{p}$ acting as the matrix

$$
\left[\begin{array}{rr}
\cos (2 \pi r / p) & -\sin (2 \pi r / p) \\
\sin (2 \pi r / p) & \cos (2 \pi r / p)
\end{array}\right],
$$

which commutes with $\left[\begin{array}{rr}0 & -1 \\ 1 & 0\end{array}\right]$. Therefore each $W_{r}$ together with $W$ itself has a natural complex structure compatible with the $C_{p}$-action, so $W \otimes_{\mathbb{R}} U$ can be viewed as a complex $C_{p}$-representation. In particular, for any $n$, as a $C_{p}$-representation,

$$
\left(\mathbb{R}^{n}\right)^{p} \cong \mathbb{R}^{n} \oplus\left(W \otimes_{\mathbb{R}} \mathbb{R}^{n}\right) \cong \mathbb{R}^{n} \oplus W^{n}
$$

where $W^{n}$ has the componentwise action, and it follows that

$$
D_{C_{p}} S^{n} \cong S^{n} \wedge E \Sigma_{p} \ltimes_{C_{p}}\left(W^{n}\right)^{\dagger},
$$

where $(-)^{\dagger}$ denotes one-point compactification of a vector space. Here $E \Sigma_{p} \ltimes_{C_{p}}$ $\left(W^{n}\right)^{\dagger}$ is the Thom spectrum of the bundle

$$
E \Sigma_{p} \ltimes_{C_{p}} W^{n} \downarrow B C_{p} .
$$

As explained in [7, Section V.2], this spectrum can be interpreted as the suspension spectrum of a truncated lens space, but the orientability of this bundle suffices for our purposes since there is a Thom isomorphism in mod $p$ homology

$$
H_{*}\left(B C_{p}\right) \cong H_{*+n(p-1)}\left(E \Sigma_{p} \ltimes_{C_{p}}\left(W^{n}\right)^{\dagger}\right) .
$$


We remark that this viewpoint is likely to be useful in investigating the kind of operations mentioned in Section 3 associated with Lubin-Tate spectra and Morava $K$-theory.

Let $z \in H^{1}\left(B C_{p}\right)$ and let $y=\beta z \in H^{2}\left(B C_{p}\right)$ be generators of

$$
H^{*}\left(B C_{p}\right)=\mathbb{F}_{p}[y] \otimes \Lambda(z) .
$$

Let $a_{n} \in H_{n}\left(B C_{p}\right)$ be dual to $z^{\varepsilon(n)} y^{\lfloor n / 2\rfloor}$, where $\varepsilon(n)=\left(1-(-1)^{n}\right) / 2$ and $\lfloor-\rfloor$ is the floor function. We will use a formula for the left coaction $\psi: H_{*}\left(B C_{p}\right) \rightarrow$ $\mathcal{A}_{*} \otimes H_{*}\left(B C_{p}\right)$, originally due to Milnor [16] (see also Boardman's account [6]).

We introduce two formal variables $t_{+}, t_{-}$in degrees $-2,-1$ respectively (so the usual graded commutativity rules apply), and defining generating series

$$
\begin{array}{ll}
a(t)=a\left(t_{+}, t_{-}\right)=\sum_{\substack{n \geqslant 1 \\
r=0,1}} a_{2 n-r} t_{+}^{n-r} t_{-}^{r}, & \\
\xi(t)=\xi\left(t_{+}\right)=\sum_{r \geqslant 0} \xi_{r} t_{+}^{p^{r}}, & \zeta(t)=\zeta\left(t_{+}\right)=\sum_{r \geqslant 0} \zeta_{r} t_{+}^{r^{r}}, \\
\tau(t)=\tau\left(t_{+}, t_{-}\right)=t_{-}+\sum_{r \geqslant 0} \tau_{r} t_{+}^{p^{r}}, & \bar{\tau}(t)=\bar{\tau}\left(t_{+}, t_{-}\right)=t_{-}+\sum_{r \geqslant 0} \bar{\tau}_{r} t_{+}^{p^{r}} .
\end{array}
$$

Notice that $\tau(t)$ and $\bar{\tau}(t)$ have odd degree and so $\tau(t)^{2}=0=\bar{\tau}(t)^{2}$. The left coaction is given by

$$
\begin{aligned}
& \psi a(t)=\sum_{\substack{n \geqslant 1 \\
r=0,1}} \psi a_{2 n-r} t_{+}^{n-r} t_{-}^{r}=a\left(\xi\left(t_{+}\right), \tau\left(t_{+}, t_{-}\right)\right) \\
& =\sum_{k \geqslant 1}\left(\xi\left(t_{+}\right)^{k} \otimes a_{2 k}-\tau\left(t_{+}, t_{-}\right) \xi\left(t_{+}\right)^{k-1} \otimes a_{2 k-1}\right) \\
& =\sum_{k \geqslant 1}\left(\xi\left(t_{+}\right)^{k} \otimes a_{2 k}-\sum_{r \geqslant 0} \tau_{r} t_{+}^{p^{r}} \xi\left(t_{+}\right)^{k-1} \otimes a_{2 k-1}+\xi\left(t_{+}\right)^{k-1} \otimes a_{2 k-1} t_{-}\right) \\
& =\sum_{k \geqslant 1}\left(\xi\left(t_{+}\right)^{k} \otimes a_{2 k}-\sum_{r \geqslant 0} \tau_{r} t_{+}^{p^{r}} \xi\left(t_{+}\right)^{k-1} \otimes a_{2 k-1}-\xi\left(t_{+}\right)^{k-1} t_{-} \otimes a_{2 k-1}\right) .
\end{aligned}
$$

Notice the effect of interchanging $t_{-}$and $a_{2 k-1}$ which disappears when we instead take the right coaction:

$$
\begin{aligned}
& \widetilde{\psi} a(t)=a\left(\zeta\left(t_{+}\right), \bar{\tau}\left(t_{+}, t_{-}\right)\right) \\
& =\sum_{k \geqslant 1}\left(a_{2 k} \otimes \zeta\left(t_{+}\right)^{k}+a_{2 k-1} \otimes \bar{\tau}\left(t_{+}, t_{-}\right) \zeta\left(t_{+}\right)^{k-1}\right) \\
& =\sum_{k \geqslant 1}\left(a_{2 k} \otimes \zeta\left(t_{+}\right)^{k}+\sum_{r \geqslant 0} a_{2 k-1} \otimes \bar{\tau}_{r} t_{+}^{p^{r}} \zeta\left(t_{+}\right)^{k-1}+a_{2 k-1} \otimes \zeta\left(t_{+}\right)^{k-1} t_{-}\right) .
\end{aligned}
$$


By comparing coefficients of monomials in $t_{+}$and $t_{-}$we obtain explicit formulae for $n \geqslant 1$ :

$$
\begin{aligned}
\psi\left(a_{2 n}\right) & =\sum_{k=1}^{n}\left[\xi\left(t_{+}\right)^{k}\right]_{t_{+}^{n}} \otimes a_{2 k}-\sum_{\substack{0 \leqslant p^{r} \leqslant n \\
1 \leqslant k \leqslant n}}\left[\tau_{r} t_{+}^{p^{r}} \xi\left(t_{+}\right)^{k-1}\right]_{t_{+}^{n}} \otimes a_{2 k-1}, \\
\psi\left(a_{2 n-1}\right) & =\sum_{k=1}^{n}\left[\xi\left(t_{+}\right)^{k-1}\right]_{t_{+}^{n-1}} \otimes a_{2 k-1},
\end{aligned}
$$

or equivalently

$$
\begin{aligned}
\widetilde{\psi}\left(a_{2 n}\right) & =\sum_{k=1}^{n} a_{2 k} \otimes\left[\zeta\left(t_{+}\right)^{k}\right]_{t_{+}^{n}}+\sum_{\substack{0 \leqslant p^{r} \leqslant n \\
1 \leqslant k \leqslant n}} a_{2 k-1} \otimes\left[\bar{\tau}_{r} t_{+}^{p^{r}} \zeta\left(t_{+}\right)^{k-1}\right]_{t_{+}^{n}}, \\
\widetilde{\psi}\left(a_{2 n-1}\right) & =\sum_{k=1}^{n} a_{2 k-1} \otimes\left[\zeta\left(t_{+}\right)^{k-1}\right]_{t_{+}^{n-1}} .
\end{aligned}
$$

We recall from [7, Chapter III] the following definitions of Dyer-Lashof operations on $x \in H_{m}(E)$. As for the prime $2, \mathrm{Q}^{r} x$ and $\beta \mathrm{Q}^{r} x$ originate on elements $H_{*}\left(D_{C_{p}} S^{m}\right)$, namely whenever $2 r \geqslant m$,

$$
\begin{gathered}
(-1)^{r} \nu(m) a_{(2 r-m)(p-1)} \otimes x_{m}^{\otimes p} \mapsto \mathrm{Q}^{r} x, \\
(-1)^{r} \nu(m) a_{(2 r-m)(p-1)-1} \otimes x_{m}^{\otimes p} \mapsto \beta \mathrm{Q}^{r} x .
\end{gathered}
$$

where

$$
\nu(m)=(-1)^{m(m-1)(p-1) / 4}(((p-1) / 2) !)^{m} .
$$

Notice that this factor does not depend on $r$.

We will use generating series in the indeterminates $t_{+}, t_{-}$for encoding actions of Dyer-Lashof operations. We set

$$
\begin{gathered}
\mathrm{Q}_{t}(-)=\mathrm{Q}_{t_{+}, t_{-}}(-)=\sum_{r} \mathrm{Q}^{r}(-) t_{+}^{r(p-1)}, \\
\beta \mathrm{Q}_{t}(-)=\beta \mathrm{Q}_{t_{+}, t_{-}}(-)=\sum_{r} \beta \mathrm{Q}^{r}(-) t_{+}^{r(p-1)-1} t_{-},
\end{gathered}
$$

where the coefficients are operators that can be applied to homology elements.

We obtain the following result on the coaction and Dyer-Lashof operations in the homology of an $H_{\infty}$ ring spectrum $E$. To ease the notation, we state it in terms of the right coaction $\widetilde{\psi}$, omitting $\otimes$ when no confusion seems likely.

Choose $\omega \in \mathbb{F}_{p^{2}}^{\times}$to be a primitive $(p-1)$-th root of -1 ; although not uniquely determined, $\omega$ gives a well defined element of the cyclic group $\mathbb{F}_{p^{2}}^{\times} / \mathbb{F}_{p}^{\times}$. We make use of the ceiling function $\lceil-\rceil$. 
Theorem 4.5. For $x \in H_{m}(E)$,

$$
\begin{aligned}
& \widetilde{\psi}\left(\mathrm{Q}_{t} x\right)=\sum_{\lceil m / 2\rceil \leqslant r} \widetilde{\psi}\left(\mathrm{Q}^{r} x\right) t_{+}^{r(p-1)} \\
& =\mathrm{Q}_{\omega \zeta\left(\omega^{-1} t_{+}\right)}(\widetilde{\psi} x) \\
& +(-1)^{m}\left(\beta \mathrm{Q}_{\omega \zeta\left(\omega^{-1} t_{+}\right), \bar{\tau}\left(\omega \zeta\left(\omega^{-1} t_{+}\right), \omega^{-1} t_{-}\right)}(\widetilde{\psi} x)-\beta \mathrm{Q}_{\omega \zeta\left(\omega^{-1} t_{+}\right), t_{-}}(\widetilde{\psi} x)\right) \\
& =\sum_{\lceil m / 2\rceil \leqslant k} \mathrm{Q}^{k}(\widetilde{\psi} x)\left(1 \otimes\left(\omega \zeta\left(\omega^{-1} t_{+}\right)\right)^{k(p-1)}\right) \\
& +\sum_{r \geqslant 0} \sum_{\lceil m / 2\rceil \leqslant \ell}(-1)^{m+r} \beta \mathrm{Q}^{\ell}(\widetilde{\psi} x)\left(1 \otimes \bar{\tau}_{r} t_{+}^{p^{r}}\left(\omega \zeta\left(\omega^{-1} t_{+}\right)\right)^{\ell(p-1)-1}\right), \\
& \widetilde{\psi}\left(\beta \mathrm{Q}_{t}(x)\right)=\sum_{\lceil m / 2\rceil \leqslant r} \widetilde{\psi}\left(\beta \mathrm{Q}^{r} x\right) t_{+}^{r(p-1)-1} t_{-} \\
& =\beta \mathrm{Q}_{\omega \zeta\left(\omega^{-1} t_{+}\right), t_{-}}(\widetilde{\psi} x)=\sum_{\lceil m / 2\rceil \leqslant s} \beta \mathrm{Q}^{s}(\widetilde{\psi} x)\left(1 \otimes\left(\omega \zeta\left(\omega^{-1} t_{+}\right)\right)^{s(p-1)-1} t_{-}\right) .
\end{aligned}
$$

Outline of proof for $m \geqslant 0$. Using the description of an extended power $D_{C_{p}} S^{m}$ as a suspension of a truncated lens space, we can pull back to $B C_{p}$. The origins of $\mathrm{Q}^{r} x, \beta \mathrm{Q}^{r} x$ then lie in the $m$-fold suspension of the elements

$(-1)^{r} a_{2(p-1) r}=\left(\omega^{-1}\right)^{r(p-1)} a_{2(p-1) r}, \quad(-1)^{r} a_{2(p-1) r-1}=\left(\omega^{-1}\right)^{r(p-1)} a_{2(p-1) r-1}$.

As the map defining the Dyer-Lashof operations factors through $D_{C_{p}} S^{m} \rightarrow D_{p} S^{m}$, the formulae follow from (4.7) and (4.8).

The case where $m<0$ can be proved using Thom spectra of virtual bundles.

The odd primary part of Steinberger's [7, Theorem III.2.2] gives the following result.

Theorem 4.6. For $r, s \geqslant 1$,

$$
\begin{aligned}
\mathrm{Q}^{\left(p^{s}-1\right) /(p-1)} \tau_{0} & =(-1)^{s} \bar{\tau}_{s}, \\
\beta \mathrm{Q}^{\left(p^{s}-1\right) /(p-1)} \tau_{0}= & (-1)^{s} \chi \xi_{s}=(-1)^{s} \zeta_{s}, \quad \beta \mathrm{Q}^{r} \tau_{0} \neq 0, \\
\mathrm{Q}^{r} \zeta_{s} & = \begin{cases}(-1)^{s} \beta \mathrm{Q}^{r+\left(p^{s}-1\right) /(p-1)} \tau_{0} & \text { if } r \equiv-1\left(\bmod p^{s}\right), \\
(-1)^{s+1} \beta \mathrm{Q}^{r+\left(p^{s}-1\right) /(p-1)} \tau_{0} & \text { if } r \equiv 0\left(\bmod p^{s}\right), \\
0 & \text { otherwise, }\end{cases} \\
\mathrm{Q}^{r} \bar{\tau}_{s} & = \begin{cases}(-1)^{s+1} \mathrm{Q}^{r+\left(p^{s}-1\right) /(p-1)} \tau_{0} & \text { if } r \equiv 0\left(\bmod p^{s}\right), \\
0 & \text { otherwise. }\end{cases}
\end{aligned}
$$

In particular,

$$
\mathrm{Q}^{p^{s}} \zeta_{s}=\zeta_{s+1}, \quad \mathrm{Q}^{p^{s}} \bar{\tau}_{s}=\bar{\tau}_{s+1}
$$


Our next result is analogous to Lemma 4.4.

Lemma 4.7. For $r \geqslant 0$ and $s \geqslant 1$,

$$
\begin{aligned}
\mathrm{Q}^{p^{r}} \tau_{r} & =\tau_{r+1}-\tau_{0} \xi_{r+1}, \\
\beta \mathrm{Q}^{p^{r}} \tau_{r} & =\xi_{r+1}, \\
\mathrm{Q}^{p^{s}} \xi_{s} & =\xi_{s+1}-\xi_{1} \xi_{s}^{p} .
\end{aligned}
$$

Proof. Conjugation is defined by the recursive formulae

$$
\begin{array}{r}
\bar{\tau}_{s}+\bar{\tau}_{s-1} \xi_{1}^{p^{s-1}}+\bar{\tau}_{s-2} \xi_{2}^{p^{s-2}}+\cdots+\bar{\tau}_{0} \xi_{s}+\tau_{s}=0 \\
\zeta_{s}+\zeta_{s-1} \xi_{1}^{p^{s-1}}+\zeta_{s-2} \xi_{2}^{p^{s-2}}+\cdots+\zeta_{1} \xi_{s-1}^{p}+\xi_{s}=0 .
\end{array}
$$

Applying $\mathrm{Q}^{p^{s}}$ to the first equation, using the Cartan formula and considering degrees carefully, we obtain

$$
\begin{aligned}
\mathrm{Q}^{p^{s}} \tau_{s} & =-\left(\mathrm{Q}^{p^{s}} \bar{\tau}_{s}+\left(\mathrm{Q}^{p^{s-1}} \bar{\tau}_{s-1}\right) \xi_{1}^{p^{s}}+\left(\mathrm{Q}^{p^{s-2}} \bar{\tau}_{s-2}\right) \xi_{2}^{p^{s-1}}+\cdots+\left(\mathrm{Q}^{1} \bar{\tau}_{0}\right) \xi_{s}^{p}\right) \\
& =-\left(\bar{\tau}_{s+1}+\bar{\tau}_{s} \xi_{1}^{p^{s}}+\bar{\tau}_{s-1} \xi_{2}^{p^{s-1}}+\cdots+\bar{\tau}_{1} \xi_{s}^{p}\right) \\
& =-\left(\bar{\tau}_{s+1}+\bar{\tau}_{s} \xi_{1}^{p^{s}}+\bar{\tau}_{s-1} \xi_{2}^{p^{s-1}}+\cdots+\bar{\tau}_{1} \xi_{s}^{p}+\bar{\tau}_{0} \xi_{s+1}+\tau_{s+1}\right)+\bar{\tau}_{0} \xi_{s+1}+\tau_{s+1} \\
& =\tau_{s+1}-\tau_{0} \xi_{s+1},
\end{aligned}
$$

and

$$
\begin{aligned}
\mathrm{Q}^{p^{s}} \xi_{s} & =-\left(\mathrm{Q}^{p^{s}} \zeta_{s}+\left(\mathrm{Q}^{p^{s-1}} \zeta_{s-1}\right) \xi_{1}^{p^{s}}+\left(\mathrm{Q}^{p^{s-2}} \zeta_{s-2}\right) \xi_{2}^{p^{s-1}}+\cdots+\left(\mathrm{Q}^{p} \zeta_{1}\right) \xi_{s}^{p^{2}}\right) \\
& =-\left(\zeta_{s+1}+\zeta_{s} \xi_{1}^{p^{s}}+\zeta_{s-1} \xi_{2}^{p^{s-1}}+\cdots+\zeta_{2} \xi_{s}^{p^{2}}\right) \\
& =-\left(\zeta_{s+1}+\zeta_{s} \xi_{1}^{p^{s}}+\zeta_{s-1} \xi_{2}^{p^{s-1}}+\cdots+\zeta_{2} \xi_{s}^{p^{2}}+\zeta_{1} \xi_{s}^{p}+\xi_{s+1}\right)+\zeta_{1} \xi_{s}^{p}+\xi_{s+1} \\
& =\xi_{s+1}-\xi_{1} \xi_{s}^{p} .
\end{aligned}
$$

We also have

$$
\beta \mathrm{Q}^{p^{s}} \tau_{s}=\beta \tau_{s+1}-\beta\left(\tau_{0} \xi_{s+1}\right)=0-\left(\beta \tau_{0}\right) \xi_{s+1}+\tau_{0}\left(\beta \xi_{s+1}\right)=\xi_{s+1},
$$

since the Bockstein $\beta$ acts on $\mathcal{A}_{*}$ by the left action of $\mathcal{A}^{*}$, i.e., if $a \in \mathcal{A}_{*}$ and $\psi(a)=\sum_{i} a_{i}^{\prime} \otimes a_{i}^{\prime \prime}$, then

$$
\beta a=\sum_{i}\left\langle\beta, \chi\left(a_{i}^{\prime}\right)\right\rangle a_{i}^{\prime \prime}
$$

where $\langle-,-\rangle$ is the dual pairing between $\mathcal{A}^{*}$ and $\mathcal{A}_{*}$. This gives $\beta \tau_{0}=-1$ as used above. 


\section{§5. Dyer-Lashof operations on the dual Steenrod algebra}

We will require more information on the action of Dyer-Lashof operations in $\mathcal{A}(p)_{*}$. In the discussion following we make use of Kochman [11] and Steinberger [7].

Let $\mathrm{R}$ be a commutative ring. Define the Newton polynomials

$$
\mathrm{N}_{n}(t)=\mathrm{N}_{n}\left(t_{1}, \ldots, t_{n}\right) \in \mathrm{R}\left[t_{1}, \ldots, t_{n}\right]
$$

recursively by setting $\mathrm{N}_{1}(t)=t_{1}$ and

$$
\mathrm{N}_{n}(t)=t_{1} \mathrm{~N}_{n-1}(t)-t_{2} \mathrm{~N}_{n-2}(t)+\cdots+(-1)^{n-2} t_{n-1} \mathrm{~N}_{1}(t)+(-1)^{n-1} n t_{n} .
$$

It is well known that for a prime $p$,

$$
\mathrm{N}_{p n}(t) \equiv \mathrm{N}_{n}(t)^{p}(\bmod p),
$$

In $\mathcal{A}(p)_{*}$, we can consider the values of these obtained by setting

$$
t_{n}=\left\{\begin{aligned}
\xi_{r} & \text { if } n=p^{r}-1 \\
0 & \text { otherwise }
\end{aligned}\right.
$$

and we denote these elements by $\mathrm{N}_{n}(\xi)$. They satisfy recurrence relations of the form

$$
\mathrm{N}_{n}(\xi)=-\xi_{1} \mathrm{~N}_{n-p+1}(\xi)-\xi_{2} \mathrm{~N}_{n-p^{2}+1}(\xi)+\cdots
$$

and in particular

$$
\begin{aligned}
\mathrm{N}_{p^{s}-1}(\xi) & =-\xi_{1} \mathrm{~N}_{p^{s}-p}(\xi)-\xi_{2} \mathrm{~N}_{p^{s}-p^{2}}(\xi)+\cdots-\xi_{s-1} \mathrm{~N}_{p^{s}-p^{s-1}}(\xi)-\left(p^{s}-1\right) \xi_{s} \\
& =-\xi_{1} \mathrm{~N}_{p^{s-1}-1}(\xi)^{p}-\xi_{2} \mathrm{~N}_{p^{s-2}-1}(\xi)^{p^{2}}+\cdots-\xi_{s-1} \mathrm{~N}_{p-1}(\xi)^{s^{s-1}}+\xi_{s} .
\end{aligned}
$$

Since $\mathrm{N}_{p-1}(\xi)=\xi_{1}=-\zeta_{1}$, it follows that the negatives $-\mathrm{N}_{p^{r}-1}(\xi)$ satisfy the same recurrence relation as the conjugates $\zeta_{r}=\chi\left(\xi_{r}\right)$, hence for each $s \geqslant 1$,

$$
\mathrm{N}_{p^{s}-1}(\xi)=-\zeta_{s} .
$$

See [1, Lemma 2.8] for a closely related result which also implies this one. We also mention another easy consequence of the recursion formula which can be verified by working modulo the ideal $\left(\xi_{i}: i \geqslant 2\right) \triangleleft \mathcal{A}(p)_{*}$.

Lemma 5.1. For any prime $p$ and any $k \geqslant 1$,

$$
\mathrm{N}_{k(p-1)}(\xi) \neq 0 \text {. }
$$

The generating series for the $(-1)^{n} \mathrm{~N}_{n}(\xi)$ satisfies the relation

$$
\left(1+\sum_{r \geqslant 1} \xi_{r} t^{p^{r}-1}\right)\left(\sum_{n \geqslant 1}(-1)^{n} \mathrm{~N}_{n}(\xi) t^{n}\right)=\sum_{r \geqslant 1} \xi_{r} t^{p^{r}-1},
$$


hence

$$
\sum_{n \geqslant 1}(-1)^{n} \mathrm{~N}_{n}(\xi) t^{n}=1-\left(1+\sum_{r \geqslant 1} \xi_{r} t^{p^{r}-1}\right)^{-1}=1-\frac{t}{\xi(t)}
$$

We will give formulae for the $\mathrm{N}_{n}(\xi)$ modulo the ideal $\left(\zeta_{j}: j \neq s\right) \triangleleft \mathcal{A}(p)_{*}$, for some fixed $s \geqslant 1$. The recursive formula for the antipode of $\mathcal{A}(p)_{*}$ gives

$$
\xi_{n s} \equiv-\zeta_{s} \xi_{(n-1) s}^{p^{s}} \bmod \left(\zeta_{j}: j \neq s\right),
$$

and an induction shows that

$$
\xi_{n s} \equiv(-1)^{n} \zeta_{s}^{\left(p^{n s}-1\right) /\left(p^{s}-1\right)} \bmod \left(\zeta_{j}: j \neq s\right) .
$$

Combining this with (5.2) we obtain

$$
\begin{aligned}
\sum_{n \geqslant 1}( & -1)^{n} \mathrm{~N}_{n}(\xi) t^{n} \\
& \equiv 1-\left(1+\sum_{r \geqslant 1}(-1)^{r} \zeta_{s}^{\left(p^{r s}-1\right) /\left(p^{s}-1\right)} t^{p^{r s}-1}\right)^{-1} \bmod \left(\zeta_{j}: j \neq s\right) .
\end{aligned}
$$

Our next result is a number-theoretic observation.

Lemma 5.2. Let $p$ be a prime and let $s \geqslant 1$. Suppose that the natural number $n$ has p-adic expansion

$$
n=n_{k} p^{k}+n_{k+1} p^{k+1}+\cdots+n_{k+\ell} p^{k+\ell}
$$

where $\ell \geqslant 0$ and $n_{k}, n_{k+\ell} \not \equiv 0(\bmod p)$. Then

$$
\begin{aligned}
&\left(\begin{array}{c}
n p^{s}-1 \\
n
\end{array}\right) \not \equiv 0(\bmod p) \\
& \Leftrightarrow n_{s+k} \leqslant n_{k}-1, n_{s+k+1} \leqslant n_{k+1}, \ldots, n_{k+\ell} \leqslant n_{k+\ell-s}
\end{aligned}
$$

Proof. The $p$-adic expansion of $n p^{s}-1$ is

$$
\begin{aligned}
n p^{s}-1= & (p-1)+(p-1) p+\cdots+(p-1) p^{s+k-1} \\
& +\left(n_{k}-1\right) p^{s+k}+n_{k+1} p^{s+k+1}+\cdots+n_{k+\ell} p^{s+k+\ell}
\end{aligned}
$$

so

$$
\left(\begin{array}{c}
n p^{s}-1 \\
n
\end{array}\right) \equiv\left(\begin{array}{c}
p-1 \\
n_{k}
\end{array}\right) \cdots\left(\begin{array}{c}
p-1 \\
n_{s+k-1}
\end{array}\right)\left(\begin{array}{c}
n_{k}-1 \\
n_{s+k}
\end{array}\right)\left(\begin{array}{c}
n_{k+1} \\
n_{s+k+1}
\end{array}\right) \cdots\left(\begin{array}{c}
n_{k+\ell-s} \\
n_{k+\ell}
\end{array}\right)(\bmod p) .
$$

This does not vanish $\bmod p$ exactly when the stated conditions hold. 
For example, when $p=2$ and $s=1$,

$$
\left(\begin{array}{c}
2 n-1 \\
n
\end{array}\right) \not \equiv 0(\bmod 2) \Leftrightarrow n=2^{k} \text { for some } k \geqslant 0 .
$$

We will use this in proving our next result.

Lemma 5.3. Let $p$ be a prime and let $s \geqslant 1$. Then

$$
\begin{aligned}
(1+ & \left.\sum_{m \geqslant 1}(-1)^{m} \zeta_{s}^{\left(p^{m s}-1\right) /\left(p^{s}-1\right)} t^{p^{m s}-1}\right)^{-1} \\
& \equiv 1+\sum_{\substack{n \in \mathbb{N} \\
\left(\begin{array}{c}
n p^{s}-1 \\
n
\end{array}\right) \neq 0(\bmod p)}}(-1)^{n+1}\left(\begin{array}{c}
n p^{s}-1 \\
n
\end{array}\right) \zeta_{s}^{n} t^{n\left(p^{s}-1\right)} \bmod \left(\zeta_{j}: j \neq s\right) .
\end{aligned}
$$

Hence

$$
\mathrm{N}_{n\left(p^{s}-1\right)}(\xi) \equiv\left(\begin{array}{c}
n p^{s}-1 \\
n
\end{array}\right) \zeta_{s}^{n} \bmod \left(\zeta_{j}: j \neq s\right),
$$

and this is non-zero precisely when the coefficients in the p-adic expansion

$$
n=n_{k} p^{k}+\cdots+n_{k+\ell} p^{k+\ell}
$$

satisfy the inequalities

$$
n_{s+k} \leqslant n_{k}-1, \quad n_{s+k+1} \leqslant n_{k+1}, \ldots, n_{k+\ell} \leqslant n_{k+\ell-s} .
$$

Proof. We will use residue calculus to determine the coefficient of $t^{n}$ of positive degree, and will denote by

$$
\oint f(z) d z=c_{-1}
$$

the coefficient of $z^{-1}$ in a meromorphic Laurent series

$$
f(z)=\sum_{k_{0} \leqslant k \in \mathbb{Z}} c_{k} z^{k} \in \mathrm{R} \llbracket z \rrbracket\left[z^{-1}\right],
$$

where $\mathrm{R}$ is any commutative ring and $k_{0} \in \mathbb{Z}$. We may apply standard rules of calculus for manipulating such expressions. For example, on changing variable by setting $z=h(w) \in \mathrm{R} \llbracket w \rrbracket\left[w^{-1}\right]$, we obtain

$$
\oint f(z) d z=\oint f(h(w)) h^{\prime}(w) d w .
$$


We may determine the coefficient of $t^{n\left(p^{s}-1\right)}$ in $t / \xi(t)$ by calculating

$$
\begin{gathered}
\oint \frac{t}{\xi(t)} \frac{d t}{t^{n\left(p^{s}-1\right)+1}}=\oint \frac{\zeta(u)}{u} \frac{d u}{\zeta(u)^{n\left(p^{s}-1\right)+1}}=\oint\left(\frac{\zeta(u)}{u}\right)^{-n\left(p^{s}-1\right)} \frac{d u}{u^{n\left(p^{s}-1\right)+1}} \\
\equiv \oint\left(1+\zeta_{s} u^{p^{s}-1}\right)^{-n\left(p^{s}-1\right)} \frac{d u}{u^{n\left(p^{s}-1\right)+1} \equiv \oint\left(1+\zeta_{s} v\right)^{-n\left(p^{s}-1\right)} \frac{(-1) d v}{v^{n+1}}} \\
\equiv-\left(\begin{array}{c}
-n\left(p^{s}-1\right) \\
n
\end{array}\right) \zeta_{s}^{n} \equiv(-1)^{n+1}\left(\begin{array}{c}
n\left(p^{s}-1\right)+(n-1) \\
n
\end{array}\right) \zeta_{s}^{n} \\
\equiv(-1)^{n+1}\left(\begin{array}{c}
n p^{s}-1 \\
n
\end{array}\right) \zeta_{s}^{n} \bmod \left(\zeta_{j}: j \neq s\right) .
\end{gathered}
$$

Now we can use Lemma 5.2 to complete the analysis of these coefficients.

To determine the Dyer-Lashof operations on the dual Steenrod algebra $\mathcal{A}_{*}$ we use results of Kochman [11], where the operations are calculated in $H_{*}\left(B O ; \mathbb{F}_{2}\right)$ and $H_{*}\left(B U ; \mathbb{F}_{p}\right)$. We remark that in each case the Thom isomorphism is known to respect the Dyer-Lashof operations, so this determines the Dyer-Lashof actions in $H_{*}\left(M O ; \mathbb{F}_{2}\right)$ and $H_{*}\left(M U ; \mathbb{F}_{p}\right)$.

For $p=2, H_{*}(M O)=H_{*}\left(M O ; \mathbb{F}_{2}\right)$ is the polynomial algebra on generators $a_{n} \in H_{n}(M O)$ which correspond to generators of $H_{*}(B O)$ coming from those in $H_{*}(B O(1))=H_{*}\left(\mathbb{R P}^{\infty}\right)$. Under the homomorphism $H_{*}(M O) \rightarrow \mathcal{A}_{*}$ induced by the orientation $\mathrm{MO} \rightarrow \mathrm{HF}_{2}$,

$$
a_{n} \mapsto \begin{cases}\xi_{s} & \text { if } n=2^{s}-1, \\ 0 & \text { otherwise. }\end{cases}
$$

The Newton polynomial $\mathrm{N}_{n}(a)=\mathrm{N}_{n}\left(a_{1}, \ldots, a_{n}\right) \in H_{n}(M O)$ corresponds to the Hopf algebra primitive generator in $H_{n}(B O)$, so [11, Corollary 35] gives

$$
\mathrm{Q}^{r} \mathrm{~N}_{n}(a)=\left(\begin{array}{l}
r-1 \\
n-1
\end{array}\right) \mathrm{N}_{n+r}(a)
$$

This yields the following formula in $\mathcal{A}_{*}$ :

$$
\mathrm{Q}^{r} \mathrm{~N}_{n}(\xi)=\left(\begin{array}{l}
r-1 \\
n-1
\end{array}\right) \mathrm{N}_{n+r}(\xi) .
$$

Using (5.1) for $p=2$, we obtain

$$
\mathrm{Q}^{r} \zeta_{s}=\left(\begin{array}{c}
r-1 \\
2^{s}-2
\end{array}\right) \mathrm{N}_{2^{s}-1+r}(\xi),
$$

and it is easy to see that

$$
\left(\begin{array}{c}
r-1 \\
2^{s}-2
\end{array}\right) \equiv 1(\bmod 2) \Leftrightarrow r \equiv 0,-1\left(\bmod 2^{s}\right) .
$$

Using Lemma 5.1, this recovers part of Steinberger's result (see our Theorem 4.2). 
For an odd prime $p, H_{*}(M U)$ is polynomial on generators $b_{n} \in H_{2 n}(M U)$ coinciding under the Thom isomorphism with generators of $H_{*}(B U)$ coming from $H_{*}(B U(1))=H_{*}\left(\mathbb{C P}^{\infty}\right)$. Under the homomorphism induced by the orientation $M U \rightarrow H \mathbb{F}_{p}$,

$$
b_{n} \mapsto \begin{cases}\xi_{s} & \text { if } n=p^{s}-1, \\ 0 & \text { otherwise, }\end{cases}
$$

and so by [11, Theorem 5], the Newton polynomial $\mathrm{N}_{n}(\xi)$ satisfies

$$
\mathrm{Q}^{r} \mathrm{~N}_{n}(\xi)=(-1)^{r+n}\left(\begin{array}{l}
r-1 \\
n-1
\end{array}\right) \mathrm{N}_{n+r(p-1)}(\xi),
$$

and using (5.1) we obtain

$$
\mathrm{Q}^{r} \zeta_{s}=(-1)^{r+1}\left(\begin{array}{c}
r-1 \\
p^{s}-2
\end{array}\right) \mathrm{N}_{p^{s}-1+r(p-1)}(\xi) .
$$

It is easy to see that

$$
\left(\begin{array}{c}
r-1 \\
p^{s}-2
\end{array}\right) \not \equiv 0(\bmod p) \Leftrightarrow r \equiv 0,-1\left(\bmod p^{s}\right),
$$

thus recovering part of Steinberger's result (see Theorem 4.6).

\section{$\S 6$. Verification of the Nishida relations}

For completeness we show how the usual Nishida relations are consequences of coaction formulae.

\section{$\S 6.1$. The case $p=2$}

First we recall that with respect to the monomial basis for $\mathcal{A}_{*}=\mathcal{A}(2)_{*}$, the dual element of $\xi_{1}^{r_{1}} \cdots \xi_{\ell}^{r_{\ell}}$ is $\mathrm{Sq}^{\left(r_{1}, \ldots, r_{\ell}\right)} \in \mathcal{A}^{*}$. The dual of $\xi_{1}^{n}$ is the Steenrod operation $\mathrm{Sq}^{(n)}=\mathrm{Sq}^{n}$, i.e.,

$$
\left\langle\mathrm{Sq}^{n} \mid \xi_{1}^{r_{1}} \cdots \xi_{\ell}^{r_{\ell}}\right\rangle= \begin{cases}1 & \text { if } \xi_{1}^{r_{1}} \cdots \xi_{\ell}^{r_{\ell}}=\xi_{1}^{n} \\ 0 & \text { otherwise. }\end{cases}
$$

In terms of the right pairing, this becomes

$$
\left\langle\zeta_{1}^{r_{1}} \cdots \zeta_{\ell}^{r_{\ell}} \mid \mathrm{Sq}^{n}\right\rangle= \begin{cases}1 & \text { if } \zeta_{1}^{r_{1}} \cdots \zeta_{\ell}^{r_{\ell}}=\zeta_{1}^{n}, \\ 0 & \text { otherwise. }\end{cases}
$$

We will work with the right coaction so the latter formulae will often be used. 
Notice that for a left $\mathcal{A}_{*}$-comodule $M_{*}$ and $x \in M_{*}$, we have

$$
\begin{aligned}
\psi(x) & =\sum_{\left(r_{1}, \ldots, r_{\ell}\right)} \xi_{1}^{r_{1}} \cdots \xi_{\ell}^{r_{\ell}} \otimes \mathrm{Sq}_{*}^{\left(r_{1}, \ldots, r_{\ell}\right)} x, \\
\widetilde{\psi}(x) & =\sum_{\left(r_{1}, \ldots, r_{\ell}\right)} \mathrm{Sq}_{*}^{\left(r_{1}, \ldots, r_{\ell}\right)} x \otimes \zeta_{1}^{r_{1}} \cdots \zeta_{\ell}^{r_{\ell}} .
\end{aligned}
$$

In particular

$$
\mathrm{Sq}_{*}^{n} x=\left\langle\mathrm{Sq}^{n} \otimes 1 \mid \psi x\right\rangle=\left\langle\widetilde{\psi} x \mid 1 \otimes \mathrm{Sq}^{n}\right\rangle .
$$

We want to determine expressions of the form $\mathrm{Sq}_{*}^{r} \mathrm{Q}^{s} x$ where $x \in H_{*}(E)$ for a commutative $S$-algebra $E$. We have

$$
\mathrm{Sq}_{*}^{r} \mathrm{Q}^{s} x=\left\langle\widetilde{\psi} \mathrm{Q}^{s} x \mid 1 \otimes \mathrm{Sq}^{r}\right\rangle,
$$

and combining these for all values of $s$ we obtain

$$
\begin{aligned}
\mathrm{Sq}_{*}^{r} \mathrm{Q}_{t} x & =\sum_{s}\left(\mathrm{Sq}_{*}^{r} \mathrm{Q}^{s} x\right) t^{s}=\left\langle\sum_{s} \widetilde{\psi} \mathrm{Q}^{s} x t^{s} \mid 1 \otimes \mathrm{Sq}^{r}\right\rangle \\
& =\left\langle\sum_{k} \mathrm{Q}^{k}(\widetilde{\psi} x) \zeta(t)^{k} \mid 1 \otimes \mathrm{Sq}^{r}\right\rangle=\left\langle\mathrm{Q}_{\zeta(t)}(\widetilde{\psi} x) \mid 1 \otimes \mathrm{Sq}^{r}\right\rangle .
\end{aligned}
$$

In the expression (6.2), applying $\mathrm{Q}_{\zeta(t)}$ to a term yields

$$
\begin{aligned}
\mathrm{Q}_{\zeta(t)}\left(\mathrm{Sq}_{*}^{\left(r_{1}, \ldots, r_{\ell}\right)} x \otimes \zeta_{1}^{r_{1}} \cdots \zeta_{\ell}^{r_{\ell}}\right) & =\mathrm{Q}_{\zeta(t)}\left(\mathrm{Sq}_{*}^{\left(r_{1}, \ldots, r_{\ell}\right)} x\right) \otimes \mathrm{Q}_{\zeta(t)}\left(\zeta_{1}^{r_{1}} \cdots \zeta_{\ell}^{r_{\ell}}\right) \\
& =\mathrm{Q}_{\zeta(t)}\left(\mathrm{Sq}_{*}^{\left(r_{1}, \ldots, r_{\ell}\right)} x\right) \otimes\left(\mathrm{Q}_{\zeta(t)} \zeta_{1}\right)^{r_{1}} \cdots\left(\mathrm{Q}_{\zeta(t)} \zeta_{\ell}\right)^{r_{\ell}},
\end{aligned}
$$

so we need to investigate the terms $\mathrm{Q}_{\zeta(t)} \zeta_{s}$. In fact for our purposes it is sufficient to know these $\bmod \left(\zeta_{j}: j>1\right)$.

Lemma 6.1. For $s \geqslant 2$,

$$
\mathrm{Q}_{t} \zeta_{s} \equiv 0 \bmod \left(\zeta_{j}: j>1\right)
$$

Proof. By (5.8) and (5.7), $\mathrm{Q}^{r} \zeta_{s} \neq 0$ only when $r \equiv 0,-1\left(\bmod 2^{s}\right)$, and then

$$
\mathrm{Q}^{r} \zeta_{s}= \begin{cases}\mathrm{N}_{1+2+\cdots+2^{s-1}+2^{k}+r_{k+1} 2^{k+1}+\cdots+2^{\ell}}(\xi) & \text { if } r \equiv 0\left(\bmod 2^{s}\right), \\ \mathrm{N}_{2+\cdots+2^{s-1}+2^{k}+r_{k+1} 2^{k+1}+\cdots+2^{\ell}}(\xi) & \text { if } r \equiv-1\left(\bmod 2^{s}\right),\end{cases}
$$

for some $k, \ell$ with $s \leqslant k \leqslant \ell$. In either case we find that $\mathrm{Q}^{r} \zeta_{s} \equiv 0 \bmod \left(\zeta_{j}: j>1\right)$ by using Lemma 5.3 (with $s=1$ ).

For the case $s=1$, we have

$$
\mathrm{Q}_{t} \zeta_{1}=\frac{1}{t}-\frac{1}{\xi(t)}+\zeta_{1}
$$

hence 


$$
\begin{aligned}
\mathrm{Q}_{\zeta(t)} \zeta_{1} & =\frac{1}{\zeta(t)}-\frac{1}{t}+\zeta_{1} \equiv \frac{1-\left(1+\zeta_{1} t\right)+\zeta_{1}\left(t+\zeta_{1} t^{2}\right)}{\left(t+\zeta_{1} t^{2}\right)} \\
& \equiv \frac{\zeta_{1}^{2} t}{\left(1+\zeta_{1} t\right)} \equiv \zeta_{1}^{2} t\left(1+\zeta_{1} t\right)^{-1} \bmod \left(\zeta_{j}: j>1\right) .
\end{aligned}
$$

So we have

$$
\begin{aligned}
\mathrm{Sq}_{*}^{r} \mathrm{Q}_{t} x & =\sum_{j \geqslant 0}\left\langle\mathrm{Q}_{\zeta(t)}\left(\mathrm{Sq}_{*}^{j} x\right)\left(\mathrm{Q}_{\zeta(t)} \zeta_{1}\right)^{j} \mid 1 \otimes \mathrm{Sq}^{r}\right\rangle \\
& =\sum_{j \geqslant 0} \sum_{k}\left\langle\zeta(t)^{k}\left(\mathrm{Q}_{\zeta(t)} \zeta_{1}\right)^{j} \mid 1 \otimes \mathrm{Sq}^{r}\right\rangle \mathrm{Q}^{k} \mathrm{Sq}_{*}^{j} x \\
& =\sum_{j \geqslant 0} \sum_{k}\left\langle\zeta_{1}^{2 j} t^{j+k}\left(1+\zeta_{1} t\right)^{k-j} \mid 1 \otimes \mathrm{Sq}^{r}\right\rangle \mathrm{Q}^{k} \mathrm{Sq}_{*}^{j} x \\
& =\sum_{j \geqslant 0} \sum_{k}\left(\begin{array}{c}
k-j \\
r-2 j
\end{array}\right) \mathrm{Q}^{k} \mathrm{Sq}_{*}^{j} x t^{r+k-j},
\end{aligned}
$$

or equivalently

$$
\mathrm{Sq}_{*}^{r} \mathrm{Q}^{n} x=\sum_{j \geqslant 0}\left(\begin{array}{c}
n-r \\
r-2 j
\end{array}\right) \mathrm{Q}^{n-r+j} \mathrm{Sq}_{*}^{j} x,
$$

which is the usual form of the Nishida relations.

\section{$\S 6.2$. The case $p$ odd}

We begin by determining formulae for Dyer-Lashof operations in $\mathcal{A}_{*}=\mathcal{A}(p)_{*} \bmod$ $\left(\zeta_{j}: j \geqslant 2\right)$. By (5.10) and (5.11) we find that for an indeterminate $t$ of degree -2 ,

$$
\begin{aligned}
& \mathrm{Q}_{t} \zeta_{s}= \sum_{k \geqslant 1}\left((-1)^{k+1}\left(\begin{array}{c}
k p^{s}-1 \\
p^{s}-2
\end{array}\right) \mathrm{N}_{\left(k p^{s}+p^{s-1}+\cdots+p+1\right)(p-1)}(\xi) t^{k p^{s}(p-1)}\right. \\
&\left.+(-1)^{k}\left(\begin{array}{c}
k p^{s}-2 \\
p^{s}-2
\end{array}\right) \mathrm{N}_{\left(k p^{s}+p^{s-1}+\cdots+p\right)(p-1)}(\xi) t^{\left(k p^{s}-1\right)(p-1)}\right) \\
& \equiv \sum_{k \geqslant 1}(-1)^{k}\left(\mathrm{~N}_{\left(k p^{s-1}+p^{s-2}+\cdots+p+1\right)(p-1)}(\xi)\right)^{p} t^{\left(k p^{s}-1\right)(p-1)} \bmod \left(\zeta_{j}: j \geqslant 2\right),
\end{aligned}
$$

where the first term in each summand vanishes thanks to Lemma 5.3. Also, when $s \geqslant 2$, Lemma 5.3 implies that

$$
\mathrm{Q}_{t} \zeta_{s} \equiv 0 \bmod \left(\zeta_{j}: j \geqslant 2\right) .
$$

When $s=1$,

$$
\begin{aligned}
\mathrm{Q}_{t} \zeta_{1} & \equiv \sum_{k \geqslant 1}(-1)^{k}\left(\mathrm{~N}_{k(p-1)}(\xi)\right)^{p} t^{(k p-1)(p-1)} \equiv t^{-(p-1)}\left(\sum_{k \geqslant 1} \mathrm{~N}_{k(p-1)}(\xi)\left(-t^{(p-1)}\right)^{k}\right)^{p} \\
& \equiv t^{-(p-1)}\left(\sum_{k \geqslant 1} \mathrm{~N}_{k(p-1)}(\xi)\left(\omega^{-1} t\right)^{k(p-1)}\right)^{p} \bmod \left(\zeta_{j}: j \geqslant 2\right),
\end{aligned}
$$


where $\omega \in \mathbb{F}_{p^{2}}^{\times}$is a primitive ( $\left.p-1\right)$-th root of -1 as introduced earlier. Using (5.2), we obtain

$$
\mathrm{Q}_{t} \zeta_{1} \equiv-\frac{1}{\left(\omega^{-1} t\right)^{p-1}}\left(1-\frac{\omega^{-1} t}{\xi\left(\omega^{-1} t\right)}\right)^{p} \bmod \left(\zeta_{j}: j \geqslant 2\right)
$$

Replacing $t$ by $\omega \zeta\left(\omega^{-1} t\right)$ gives another useful formula:

$$
\begin{aligned}
\mathrm{Q}_{\omega \zeta\left(\omega^{-1} t\right)} \zeta_{1} & \equiv-\frac{1}{\zeta\left(\omega^{-1} t\right)^{p-1}}\left(1-\frac{\zeta\left(\omega^{-1} t\right)}{\omega^{-1} t}\right)^{p} \equiv \zeta_{1}^{p} t^{(p-1)^{2}}\left(1-\zeta_{1} t^{p-1}\right)^{1-p} \\
& \equiv \sum_{k \geqslant 0}\left(\begin{array}{c}
p-2+k \\
k
\end{array}\right) \zeta_{1}^{p+k} t^{(p+k-1)(p-1)} \bmod \left(\zeta_{j}: j \geqslant 2\right) .
\end{aligned}
$$

Now we follow a similar line of argument to that for the case $p=2$ above. We recall that $\mathcal{A}(p)_{*}$ has a basis consisting of monomials

$$
\xi_{1}^{r_{1}} \cdots \xi_{k}^{r_{k}} \tau_{0}^{e_{0}} \cdots \tau_{\ell}^{e_{\ell}}
$$

where $e_{i}=0,1$ and $r_{i} \geqslant 0$. In $\mathcal{A}(p)^{*}$, the dual basis element is $\mathcal{P}^{\left(r_{1}, \ldots, r_{k} ; e_{0}, \ldots, e_{\ell}\right)}$. In particular, $\mathcal{P}^{(r)}=\mathcal{P}^{r}$ is the reduced power operation.

We want to determine the series $\mathcal{P}_{*}^{r} \mathrm{Q}_{t} x$, and this turns out to be given by

$$
\begin{aligned}
\mathcal{P}_{*}^{r} \mathrm{Q}_{t} x & =\sum_{j \geqslant 0}\left\langle\mathrm{Q}_{\omega \zeta\left(\omega^{-1} t\right)}\left(\mathcal{P}_{*}^{j} x\right)\left(\mathrm{Q}_{\omega \zeta\left(\omega^{-1} t\right)} \zeta_{1}\right)^{j} \mid \mathcal{P}^{r}\right\rangle \\
& =\sum_{j \geqslant 0} \sum_{k}\left\langle\left(\omega \zeta\left(\omega^{-1} t\right)\right)^{k(p-1)}\left(\mathrm{Q}_{\omega \zeta\left(\omega^{-1} t\right)} \zeta_{1}\right)^{j} \mid \mathcal{P}^{r}\right\rangle \mathrm{Q}^{k} \mathcal{P}_{*}^{j} x \\
& =\sum_{j \geqslant 0} \sum_{k} t^{(k+j(p-1))(p-1)}\left\langle\zeta_{1}^{j p}\left(1-\zeta_{1} t^{p-1}\right)^{(k-j)(p-1)} \mid \mathcal{P}^{r}\right\rangle \mathrm{Q}^{k} \mathcal{P}_{*}^{j} x \\
& =\sum_{j \geqslant 0} \sum_{k} t^{(k+j(p-1))(p-1)}(-1)^{r-j p}\left(\begin{array}{c}
(k-j)(p-1) \\
r-j p
\end{array}\right) t^{(r-j p)(p-1)} \mathrm{Q}^{k} \mathcal{P}_{*}^{j} x \\
& =\sum_{j \geqslant 0} \sum_{k}(-1)^{j+r} t^{(k-j+r)(p-1)}\left(\begin{array}{c}
(k-j)(p-1) \\
r-j p
\end{array}\right) \mathrm{Q}^{k} \mathcal{P}_{*}^{j} x .
\end{aligned}
$$

Taking the coefficient of $t^{s(p-1)}$ by putting $k=s-r+j$ we obtain

$$
\mathcal{P}_{*}^{r} \mathrm{Q}^{s} x=\sum_{j \geqslant 0}(-1)^{r+j}\left(\begin{array}{c}
(s-r)(p-1) \\
r-j p
\end{array}\right) \mathrm{Q}^{s-r+j} \mathcal{P}_{*}^{j} x,
$$

which is the usual form of the Nishida relations.

We leave the interested reader to perform a similar verification of the Nishida relations for $\mathcal{P}_{*}^{r} \beta \mathrm{Q}^{s}$. 


\section{$\S 7$. Working modulo squares and Milnor primitives}

In this section we work at the prime 2, but there are analogous results at odd primes. Let $E$ be a commutative $S$-algebra.

As another example of the utility of our methods, we will investigate the induced coaction

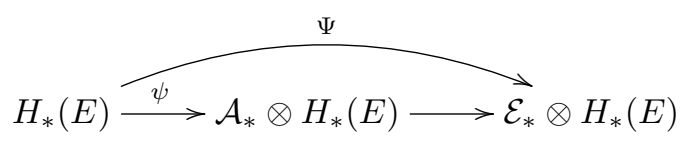

where

$$
\mathcal{E}_{*}=\mathcal{A}_{*} / / \mathcal{A}_{*}^{(2)}=\mathcal{A}_{*} /\left(\zeta_{s}^{2}: s \geqslant 1\right)
$$

is the exterior quotient Hopf algebra dual to the Hopf subalgebra of $\mathcal{A}^{*}$ generated by the Milnor primitives $\mathrm{q}^{r} \in \mathcal{A}^{2^{r+1}-1}$ recursively defined by setting $\mathrm{q}^{0}=\mathrm{Sq}^{1}$ and for $r \geqslant 1$,

$$
\mathrm{q}^{r}=\left[\mathrm{q}^{r-1}, \mathrm{Sq}^{2^{r}}\right]=\mathrm{q}^{r-1} \mathrm{Sq}^{2^{r}}+\mathrm{Sq}^{2^{r}} \mathrm{q}^{r-1}
$$

To avoid cumbersome notation, we will write $u \stackrel{\circ}{=}$ in place of $u \equiv v \bmod$ $\left(\zeta_{s}^{2}: s \geqslant 1\right)$ when working with the quotient $\operatorname{ring} \mathcal{E}_{*}$, and identify elements of $\mathcal{A}_{*}$ with their residue classes.

As with $\psi$, there is a corresponding right coaction

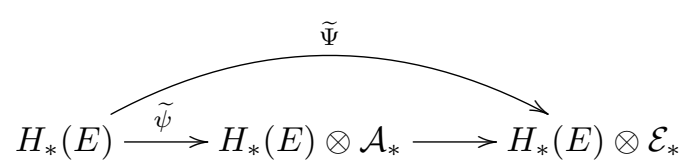

Our interest is in the general form of the right coaction on elements of the form $\mathrm{Q}^{I} z$, or equivalently in $\mathrm{q}_{*}^{r} \mathrm{Q}^{I} z$ for $r \geqslant 0$. Of course it is well known that

$$
\mathrm{q}_{*}^{0} \mathrm{Q}^{a} z=\mathrm{Sq}_{*}^{1} \mathrm{Q}^{a} z=(a+1) \mathrm{Q}^{a-1} z .
$$

Using the monomial basis in the residue classes $\bar{\xi}_{i}$ of the $\xi_{i}, \mathrm{q}^{r}$ is dual to the residue class of

$$
\xi_{r+1}=\chi\left(\zeta_{r+1}\right)=\zeta_{1} \xi_{r}^{2}+\cdots+\zeta_{r} \xi_{1}^{2^{r}}+\zeta_{r+1} \equiv \zeta_{r+1} \bmod \left(\zeta_{i}^{2}: i \geqslant 1\right) .
$$

Hence $\xi_{r+1} \stackrel{\circ}{=} \zeta_{r+1}$ is primitive in the quotient Hopf algebra $\mathcal{E}_{*}$. To calculate $\mathrm{q}_{*}^{r} w$ we may use the formulae

$$
\mathrm{q}_{*}^{r} w=\left\langle\mathrm{q}^{r} \otimes 1 \mid \Psi w\right\rangle=\left\langle\widetilde{\Psi} w \mid 1 \otimes \mathrm{q}^{r}\right\rangle .
$$

It is clear that the action of the Dyer-Lashof operations descends from $\mathcal{A}_{*}$ to the quotient $\mathcal{E}_{*}$. We start by determining the image of $\mathrm{Q}^{r} \zeta_{s}$ in $\mathcal{E}_{*}$.

Lemma 7.1. For $s \geqslant 1$ and $r \geqslant s$, we have

$$
\mathrm{Q}^{r} \zeta_{s} \stackrel{\circ}{=} \begin{cases}\zeta_{s+m} & \text { if } r=2^{s+m}-2^{s}, \\ 0 & \text { otherwise. }\end{cases}
$$


Proof. Using (5.7) and (5.8), it suffices to consider the cases $r=2^{s} k, 2^{s} k-1$. The Newton recurrence formula gives

$$
\begin{aligned}
\mathrm{Q}^{2^{s} k} \zeta_{s} & =\mathrm{N}_{2^{s}(k+1)-1}(\xi)=\xi_{1} \mathrm{~N}_{2^{s}(k+1)-2}(\xi)+\xi_{2} \mathrm{~N}_{2^{s}(k+1)-4}(\xi)+\cdots \\
& =\xi_{1} \mathrm{~N}_{2^{s-1}(k+1)-1}(\xi)^{2}+\xi_{2} \mathrm{~N}_{2^{s-2}(k+1)-1}(\xi)^{4}+\cdots
\end{aligned}
$$

and this is $0 \bmod \left(\zeta_{i}^{2}: i \geqslant 1\right)$ unless $2^{s}(k+1)=2^{s+m}$, i.e., $k=2^{m}-1$, and then

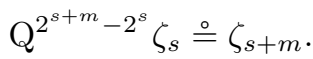

Also,

$$
\mathrm{Q}^{2^{s} k-1} \zeta_{s}=\mathrm{N}_{2^{s}(k+1)-2}(\xi)=\mathrm{N}_{2^{s-1}(k+1)-1}(\xi)^{2} \stackrel{\circ}{=} .
$$

Using the notation

$$
\Xi(r)=\sum_{r+1 \leqslant k} \zeta_{k} t^{2^{k}-2^{r}}, \quad \Xi(r, s)=\Xi(r)-\Xi(s)=\sum_{r+1 \leqslant k \leqslant s} \zeta_{k} t^{2^{k}-2^{r}},
$$

where $0 \leqslant r<s$, we obtain the following succinct formula:

$$
\mathrm{Q}_{t} \zeta_{s} \doteq \sum_{s+1 \leqslant k} \zeta_{k} t^{2^{k}-2^{s}}=\Xi(s) .
$$

If $s \geqslant 1$

$$
\Xi(s)^{2} \stackrel{\circ}{=},
$$

hence when $s_{1}<s_{2}$

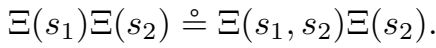

More generally, if $s_{1}<s_{2}<\cdots<s_{\ell}$, then

$$
\begin{aligned}
& \mathrm{Q}_{t}\left(\zeta_{s_{1}} \zeta_{s_{2}} \cdots \zeta_{s_{\ell}}\right)=\mathrm{Q}_{t}\left(\zeta_{s_{1}}\right) \mathrm{Q}_{t}\left(\zeta_{s_{2}}\right) \cdots \mathrm{Q}_{t}\left(\zeta_{s_{\ell}}\right)
\end{aligned}
$$

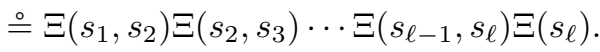

Now we can give a formula for the $\mathcal{E}_{*}$-coaction.

Proposition 7.2. If $z \in H_{n}(E)$, then

$$
\widetilde{\Psi} \mathrm{Q}_{t} z \stackrel{\circ}{=} \mathrm{Q}_{t}(\widetilde{\Psi} z)+\sum_{a \geqslant n} \sum_{j \geqslant 1}(a+1) \mathrm{Q}^{a-2^{j}+1}(\widetilde{\Psi} z) \zeta_{j} t^{a} .
$$

Equivalently, for each $a \geqslant n$,

$$
\widetilde{\Psi} \mathrm{Q}^{a} z \doteq \mathrm{Q}^{a}(\widetilde{\Psi} z)+(a+1) \sum_{j \geqslant 1} \mathrm{Q}^{a-2^{j}+1}(\widetilde{\Psi} z) \zeta_{j} .
$$


Proof. This follows from the calculation

$$
\begin{aligned}
& \widetilde{\Psi} \mathrm{Q}^{a} z \stackrel{\circ}{=} \sum_{n \leqslant k \leqslant a} \mathrm{Q}^{k}(\widetilde{\Psi} z)\left[(1+\Xi(0))^{k}\right]_{t^{a-k}} \\
& \stackrel{\circ}{=}(\widetilde{\Psi} z)+\sum_{n \leqslant k \leqslant a-1} k \mathrm{Q}^{k}(\widetilde{\Psi} z)[\Xi(0)]_{t^{a-k}}
\end{aligned}
$$

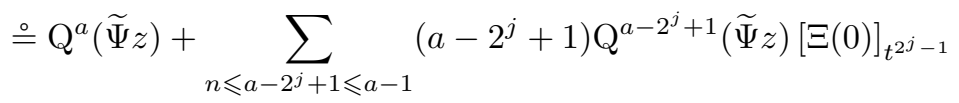

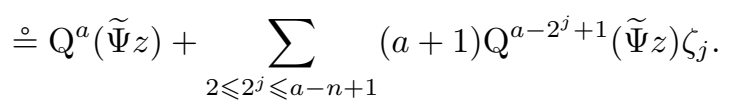

We can use this to derive formulae for the action of the Milnor primitives.

Proposition 7.3. If $z \in H_{n}(E), s \geqslant 0$ and $a>n$, then

$$
\mathrm{q}_{*}^{s} \mathrm{Q}^{a} z=(a+1) \mathrm{Q}^{a-2^{s+1}+1} z+\sum_{0 \leqslant r \leqslant s-1} \mathrm{Q}^{a-2^{s+1}+2^{r+1}}\left(\mathrm{q}_{*}^{r} z\right) .
$$

Proof. We can determine $\mathrm{q}_{*}^{s} \mathrm{Q}^{a} z$ using the inner product, i.e.,

$$
\mathrm{q}_{*}^{s} \mathrm{Q}^{a} z=\left\langle\widetilde{\Psi} \mathrm{Q}^{a} z \mid 1 \otimes \mathrm{q}^{s}\right\rangle
$$

By Proposition 7.2, we have

$$
\mathrm{q}_{*}^{s} \mathrm{Q}^{a} z=\left\langle\mathrm{Q}^{a}(\widetilde{\Psi} z) \mid 1 \otimes \mathrm{q}^{s}\right\rangle+(a+1) \mathrm{Q}^{a-2^{s+1}+1} z .
$$

To analyse $\left\langle\mathrm{Q}^{a}(\widetilde{\Psi} z) \mid 1 \otimes \mathrm{q}^{s}\right\rangle$, we note that only the term in $\mathrm{Q}^{a}(\widetilde{\Psi} z)$ of form $(?) \otimes$ $\zeta_{s+1}$ can provide a non-zero contribution, while in $\widetilde{\Psi} z$ any term of form (?) $\otimes$ $\zeta_{i_{1}} \cdots \zeta_{i_{\ell}}$ with $\ell>1$ contributes zero. Since $\mathrm{Q}^{2^{s+1}-2^{r+1}}\left(\zeta_{r+1}\right) \stackrel{\circ}{=} \zeta_{s+1}$ we must have

$$
\begin{aligned}
\left\langle\mathrm{Q}^{a}(\widetilde{\Psi} z) \mid 1 \otimes \mathrm{q}^{s}\right\rangle & =\sum_{r}\left\langle\mathrm{Q}^{a}\left(\mathrm{q}_{*}^{r} z \otimes \zeta_{r}\right) \mid 1 \otimes \mathrm{q}^{s}\right\rangle \\
& =\sum_{r}\left\langle\mathrm{Q}^{a-2^{s+1}+2^{r+1}}\left(\mathrm{q}_{*}^{r} z\right) \otimes \zeta_{s+1} \mid 1 \otimes \mathrm{q}^{s}\right\rangle \\
& =\sum_{r} \mathrm{Q}^{a-2^{s+1}+2^{r+1}}\left(\mathrm{q}_{*}^{r} z\right) .
\end{aligned}
$$

This result is useful when calculating with iterated Dyer-Lashof operations. For example,

$$
\begin{aligned}
\mathrm{q}_{*}^{1} \mathrm{Q}^{a} z & =(a+1) \mathrm{Q}^{a-3} z+\mathrm{Q}^{a-2}\left(\mathrm{q}_{*}^{0} z\right), \\
\mathrm{q}_{*}^{1} \mathrm{Q}^{a} \mathrm{Q}^{b} z & =(a+1) \mathrm{Q}^{a-3} \mathrm{Q}^{b} z+(b+1) \mathrm{Q}^{a-2} \mathrm{Q}^{b-1} z .
\end{aligned}
$$

In general, $\mathrm{q}_{*}^{s} \mathrm{Q}^{a_{1}} \cdots \mathrm{Q}^{a_{s+1}} z$ does not depend on the coaction on $z$. 


\section{Part III}

\section{Free commutative $S$-algebras}

\section{$\S 8$. Free commutative $S$-algebras and their homology}

Following [10] we work in the model categories of left $S$-modules $\mathscr{M}=\mathscr{M}_{S}$ and commutative $S$-algebras $\mathscr{C}=\mathscr{C}_{S}$. The latter are the commutative monoids in $\mathscr{M}$. The forgetful functor $\mathbb{U}: \mathscr{C} \rightarrow \mathscr{M}$ has a left adjoint $\mathbb{P}: \mathscr{M} \rightarrow \mathscr{C}$, the free commutative $S$-algebra functor, giving a Quillen adjunction:

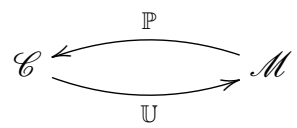

For an $S$-module $X$,

$$
\mathbb{P} X=\bigvee_{j \geqslant 0} X^{(j)} / \Sigma_{j},
$$

where $X^{(j)}=X \wedge \cdots \wedge X$ is the $j$-fold smash power with its evident $\Sigma_{j}$-action, and $X^{(j)} / \Sigma_{j}$ is the orbit spectrum. When $X$ is cofibrant, the natural map $D_{j} X \rightarrow$ $X^{(j)} / \Sigma_{j}$ is a weak equivalence, hence there is a weak equivalence

$$
\bigvee_{j \geqslant 0} D_{j} X \stackrel{\sim}{\rightarrow} \mathbb{P} X .
$$

The $\bmod p$ homology of extended powers $D_{n} X$ has been studied extensively, and the answer is expressible in terms of a free algebra construction. Recently, Kuhn \& McCarty [14] gave an explicit description for the prime 2, and we adopt a similar viewpoint. Older references of relevance are May [15], McClure [7, Theorem IX.2.1], and Kuhn [13]. In keeping with our emphasis on coactions and comodule structures, we phrase this in terms of the dual Steenrod algebra, thus avoiding the local finiteness condition for actions of the Steenrod algebra.

Fix a prime $p$ and let $\mathcal{A}_{*}=\mathcal{A}(p)_{*}$. We adopt the following notation.

- $\operatorname{Comod}_{\mathcal{A}_{*}}$ is the category of $\mathbb{Z}$-graded right $\mathcal{A}_{*}$-comodules, where we denote the coaction by $\Psi: M_{*} \rightarrow M_{*} \otimes \mathcal{A}_{*}$.

- Vect ${ }^{\mathrm{DL}}$ is the category of graded $\mathbb{F}_{p^{-}}$vector spaces $V_{*}$ equipped with actions of Dyer-Lashof operations $\mathrm{Q}^{r}: V_{*} \rightarrow V_{*+2(p-1) r}$ and $\beta \mathrm{Q}^{r}: V_{*} \rightarrow V_{*+2(p-1) r-1}$ (when $p=2, \mathrm{Q}^{r}: V_{*} \rightarrow V_{*+r}$ ) subject to the Adem relations and the unstable condition $\mathrm{Q}^{r} v=0$ if $2 r<|v|$ (when $p=2, \mathrm{Q}^{r} v=0$ if $r<|v|$ ).

- $\operatorname{Comod}_{\mathcal{A}_{*}}^{\mathrm{DL}}$ is the full subcategory of $\operatorname{Comod}_{\mathcal{A}_{*}} \cap \operatorname{Vect}^{\mathrm{DL}}$ which consists of right $\mathcal{A}_{*}$-comodules with Dyer-Lashof action that satisfies the formulae of Theorem 4.5 when $p$ is odd, or Theorem 4.1 when $p=2$. 
The free algebra $\mathbb{P} X$ has a natural homotopy coproduct $\Delta: \mathbb{P} X \rightarrow \mathbb{P} X \wedge \mathbb{P} X$ induced by the pinch map $X \rightarrow X \vee X$. The induced homomorphism

$$
\Delta_{*}: H_{*}(\mathbb{P} X) \rightarrow H_{*}(\mathbb{P} X) \otimes H_{*}(\mathbb{P} X)
$$

turns $H_{*}(\mathbb{P} X)=H_{*}\left(\mathbb{P} X ; \mathbb{F}_{p}\right)$ into a cocommutative coalgebra, and so $H_{*}(\mathbb{P} X)$ is a bicommutative Hopf algebra. This structure is discussed in detail in $[14$, Section 2.3] at least for the prime 2. The component maps of $\Delta$ are transfers associated to inclusions of block subgroups $\Sigma_{r} \times \Sigma_{s} \leqslant \Sigma_{r+s}$ and the Dyer-Lashof operations on $H_{*}(\mathbb{P} X)$ satisfy a Cartan formula making it a bicommutative $\mathcal{A}_{*}$-comodule Hopf algebra with Dyer-Lashof action satisfying the restriction condition $\mathrm{Q}^{|x| / 2} x=x^{p}$ if $|x|$ is even (and $\mathrm{Q}^{|x|} x=x^{2}$ if $p=2$ ). We denote the category of all such bicommutative Hopf algebras by $\operatorname{HopfAlg}^{\mathcal{A}_{*}, \text { DL }}$.

There are two algebraic free functors that are relevant here.

- The left adjoint

$$
\mathbf{R}: \operatorname{Comod}_{\mathcal{A}_{*}} \rightarrow \operatorname{Comod}_{\mathcal{A}_{*}}^{\mathrm{DL}}
$$

of the forgetful functor $\operatorname{Comod}_{\mathcal{A}_{*}}^{\mathrm{DL}} \rightarrow \operatorname{Comod}_{\mathcal{A}_{*}}$; this is a coproduct $\mathbf{R}=$ $\bigoplus_{s} \mathbf{R}_{s}$ where the summand $\mathbf{R}_{s}$ is expressed in terms of Dyer-Lashof words of length $s$.

- The left adjoint

$$
\mathrm{U}: \operatorname{Comod}_{\mathcal{A}_{*}}^{\mathrm{DL}} \rightarrow \operatorname{HopfAlg}^{\mathcal{A}_{*}, \mathrm{DL}}
$$

of the coalgebra primitives functor $\operatorname{Pr}: \operatorname{HopfAlg}^{\mathcal{A}_{*}, \mathrm{DL}} \rightarrow \operatorname{Comod}_{\mathcal{A}_{*}}^{\mathrm{DL}}$; this involves the free graded commutative algebra functor with additional relations coming from the restriction condition.

The structure of $H_{*}(\mathbb{P} X)$ is given by the next result.

Theorem 8.1. If $X$ is cofibrant, then in HopfAlg A $^{\mathcal{A}_{*}, \mathrm{DL}}$ there is a natural isomorphism

$$
\mathbf{U}\left(\mathbf{R}\left(H_{*}(X)\right)\right) \cong H_{*}(\mathbb{P} X)
$$

Of course this is an abstract version of a description in terms of a free algebra on admissible Dyer-Lashof monomials applied to elements of $H_{*}(X)$ with suitable excess conditions; see [2] for details.

\section{§9. Sample calculations for $p=2$}

In this section we take $p=2$, and assume that all spectra are localised at 2 . 
Consider the commutative $S$-algebra $S / / 2$ obtained as the pushout in the diagram of commutative $S$-algebras

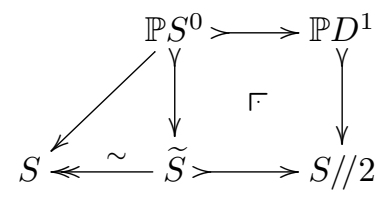

in which $S^{0} \stackrel{\sim}{\rightarrow} S$ is the functorial cofibrant replacement of $S$ as an $S$-module, $S^{0} \rightarrow D^{1} \sim *$ is the functorial cofibrant replacement for the collapse map $S^{0} \rightarrow *$, the diagonal map is induced from a realisation of the degree 2 map $S^{0} \rightarrow S$, and $\widetilde{S}$ is defined using the functorial factorisation in the model category $\mathscr{C}_{S}$. It follows that $S / / 2$ is cofibrant in $\mathscr{C}_{S}$, and furthermore there is an isomorphism of commutative $S$-algebras

$$
S / / 2 \cong \widetilde{S} \wedge_{\mathbb{P} S^{0}} \mathbb{P} D^{1} .
$$

This description allows a calculation of homology using the Künneth spectral sequence. Since the degree 2 map induces the trivial map in mod 2 ordinary homology, we can determine $H_{*}(S / / 2)=H_{*}\left(S / / 2 ; \mathbb{F}_{2}\right)$ with the aid of [3, Theorem 1.7]. The answer is

$$
H_{*}(S / / 2)=\mathbb{F}_{2}\left[\mathrm{Q}^{I} x_{1}: I \text { admissible, } \operatorname{exc}(I)>1\right],
$$

where $x_{1} \in H_{1}(S / / 2)$ satisfies $\mathrm{Sq}_{*}^{1} x_{1}=1$.

Our formulae for the right coaction give

$$
\begin{aligned}
\widetilde{\psi} \mathrm{Q}^{r} x_{1} & =\sum_{1 \leqslant k \leqslant r} \mathrm{Q}^{k}\left(x_{1} \otimes 1+1 \otimes \zeta_{1}\right)\left[1 \otimes \zeta(t)^{k}\right]_{t^{r}} \\
& =\sum_{1 \leqslant k \leqslant r}\left[\mathrm{Q}^{k} x_{1} \otimes \zeta(t)^{k}+1 \otimes\left(\mathrm{Q}^{k} \zeta_{1}\right) \zeta(t)^{k}\right]_{t^{r}}
\end{aligned}
$$

For example

$$
\begin{aligned}
& \widetilde{\psi} \mathrm{Q}^{2} x_{1}=\mathrm{Q}^{2} x_{1} \otimes 1+x_{1}^{2} \otimes \zeta_{1}+1 \otimes\left(\zeta_{1}^{3}+\zeta_{2}\right)=\mathrm{Q}^{2} x_{1} \otimes 1+x_{1}^{2} \otimes \zeta_{1}+1 \otimes \xi_{2}, \\
& \widetilde{\psi} \mathrm{Q}^{3} x_{1}=\mathrm{Q}^{3} x_{1} \otimes 1+1 \otimes \mathrm{Q}^{3} \zeta_{1}=\mathrm{Q}^{3} x_{1} \otimes 1+1 \otimes \zeta_{1}^{4},
\end{aligned}
$$

which give

$$
\psi \mathrm{Q}^{2} x_{1}=1 \otimes \mathrm{Q}^{2} x_{1}+\zeta_{1} \otimes x_{1}^{2}+\zeta_{2} \otimes 1, \quad \psi \mathrm{Q}^{3} x_{1}=1 \otimes \mathrm{Q}^{3} x_{1}+\zeta_{1}^{4} \otimes 1 .
$$

Using ideas of $[18,19]$ we will give a description of $H_{*}(S / / 2)$ as an extended $\mathcal{A}_{*}$-comodule algebra, which then gives an explicit description of $S / / 2$ as a wedge of suspensions of $\mathrm{HF}_{2}$. 
First we specify some elements, namely for $s \geqslant 1$,

$$
X_{s}=\mathrm{Q}^{2^{s}} X_{s-1}=\mathrm{Q}^{2^{s}} \mathrm{Q}^{2^{s-1}} \cdots \mathrm{Q}^{2} x_{1},
$$

where $X_{0}=x_{1}$. Notice that the degree of $X_{s}$ is $\left|X_{s}\right|=2^{s+1}-1$.

Definition 9.1. When considering an element of the form $\mathrm{Q}^{I} x$, we will say that it is strictly allowable if $I$ is admissible and $\operatorname{exc}(I)>|x|$.

This is more stringent than the usual notion of allowable where only $\operatorname{exc}(I) \geqslant$ $|x|$ is required.

Lemma 9.2. The Dyer-Lashof monomial $\mathrm{Q}^{r} X_{s}$ is only strictly allowable if $r=2^{s+1}$.

Proof. If $\mathrm{Q}^{r} X_{s}$ is admissible then $r \leqslant 2^{s+1}$, while the excess condition holds only if $r>2^{s+1}-1$.

Next we consider the coaction on these elements.

Proposition 9.3. In the ring $H_{*}(S / / 2)$, the sequence $X_{0}, X_{1}, X_{2}, \ldots$ is regular, and generates an ideal $J=\left(X_{s}: s \geqslant 0\right) \triangleleft H_{*}(S / / 2)$. The left coaction on $X_{s}$ is given by

$$
\begin{aligned}
\psi X_{s} & =1 \otimes X_{s}+\zeta_{1} \otimes X_{s-1}^{2}+\zeta_{2} \otimes X_{s-2}^{2^{2}}+\cdots+\zeta_{s} \otimes X_{0}^{2^{s}}+\zeta_{s+1} \otimes 1 \\
& \equiv \zeta_{s+1} \otimes 1 \bmod J
\end{aligned}
$$

Proof. We begin with the formula

$$
\widetilde{\psi}\left(X_{0}\right)=X_{0} \otimes 1+1 \otimes \zeta_{1}=X_{0} \otimes 1+1 \otimes \xi_{1} .
$$

We will verify by induction on $s$ that

$$
\widetilde{\psi} X_{s}=X_{s} \otimes 1+X_{s-1}^{2} \otimes \xi_{1}+X_{s-2}^{2^{2}} \otimes \xi_{2}+\cdots+X_{0}^{2^{s}} \otimes \xi_{s}+1 \otimes \xi_{s+1} .
$$

So assume that this holds for some $s \geqslant 0$. We have

$$
\begin{aligned}
\widetilde{\psi} X_{s+1}= & \widetilde{\psi} \mathrm{Q}^{2^{s+1}} X_{s}=\left(\mathrm{Q}^{2^{s+1}-1} \widetilde{\psi} X_{s}\right)\left(1 \otimes \xi_{1}\right)+\mathrm{Q}^{2^{s+1}} \widetilde{\psi} X_{s} \\
= & X_{s}^{2} \otimes \xi_{1}+X_{s-1}^{2^{2}} \otimes \xi_{1}^{3}+X_{s-2}^{2^{3}} \otimes \xi_{2}^{2} \xi_{1} \\
& +\cdots+X_{0}^{2^{s+1}} \otimes \xi_{s}^{2} \xi_{1}+1 \otimes \xi_{s+1}^{2} \xi_{1}+X_{s+1} \otimes 1 \\
& +\mathrm{Q}^{2^{s+1}-2}\left(X_{s-1}^{2}\right) \otimes \mathrm{Q}^{2} \xi_{1}+\mathrm{Q}^{2^{s+1}-2^{2}}\left(X_{s-2}^{2^{2}}\right) \otimes \mathrm{Q}^{2^{2}} \xi_{2} \\
& +\cdots+\mathrm{Q}^{2^{s+1}-2^{s}}\left(X_{0}^{2^{s}}\right) \otimes \mathrm{Q}^{2^{s}} \xi_{2}+1 \otimes \mathrm{Q}^{2^{s+1}} \xi_{s+1}
\end{aligned}
$$




$$
\begin{aligned}
= & X_{s}^{2} \otimes \xi_{1}+X_{s-1}^{2^{2}} \otimes \xi_{1}^{3}+X_{s-2}^{2^{3}} \otimes \xi_{2}^{2} \xi_{1}+\cdots+X_{0}^{2^{s+1}} \otimes \xi_{s}^{2} \xi_{1} \\
& +1 \otimes \xi_{s+1}^{2} \xi_{1}+X_{s+1} \otimes 1+X_{s-1}^{2^{2}} \otimes \mathrm{Q}^{2} \xi_{1}+X_{s-2}^{2^{3}} \otimes \mathrm{Q}^{2^{2}} \xi_{2} \\
& +\cdots+X_{0}^{2^{s+1}} \otimes \mathrm{Q}^{2^{s}} \xi_{s}+1 \otimes \mathrm{Q}^{2^{s+1}} \xi_{s+1} \\
= & X_{s+1} \otimes 1+X_{s}^{2} \otimes \xi_{1}+X_{s-1}^{2^{2}} \otimes\left(\xi_{1}^{3}+\mathrm{Q}^{2} \xi_{1}\right)+X_{s-2}^{2^{3}} \otimes\left(\xi_{2}^{2} \xi_{1}+\mathrm{Q}^{2^{2}} \xi_{2}\right) \\
& +\cdots+X_{0}^{2^{s+1}} \otimes\left(\xi_{s}^{2} \xi_{1}+\mathrm{Q}^{2^{s}} \xi_{s}\right)+1 \otimes\left(\xi_{s+1}^{2} \xi_{1}+\mathrm{Q}^{2^{s+1}} \xi_{s+1}\right) \\
= & X_{s+1} \otimes 1+X_{s}^{2} \otimes \xi_{1}+X_{s-1}^{2^{2}} \otimes \xi_{2}+X_{s-2}^{2^{3}} \otimes \xi_{3} \\
& +\cdots+X_{0}^{2^{s+1}} \otimes \xi_{s+1}+1 \otimes \xi_{s+2},
\end{aligned}
$$

where we make use of Lemma 4.4 in the last step.

Now consider the following composition of left $\mathcal{A}_{*}$-comodule algebra homomorphisms:

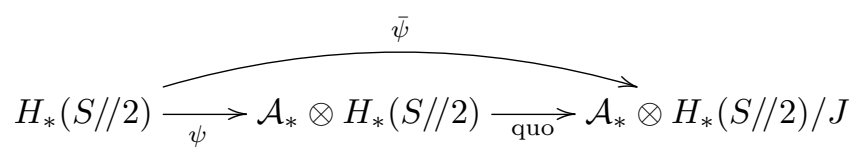

where the second and third terms are extended left comodules. By Proposition 9.3 this composition is an isomorphism of comodule algebras

$$
H_{*}(S / / 2) \stackrel{\bar{\psi}}{\cong} \mathcal{A}_{*} \otimes H_{*}(S / / 2) / J
$$

and there is a polynomial subalgebra $P_{*} \subseteq H_{*}(S / / 2)$ with $\bar{\psi} P_{*}=\mathbb{F}_{2} \otimes H_{*}(S / / 2) / J$. A standard argument shows that

$$
\pi_{*}(S / / 2) \cong P_{*}=\operatorname{Ext}_{\mathcal{A}_{*}}^{0, *}\left(\mathbb{F}_{2}, H_{*}(S / / 2)\right) \subseteq H_{*}(S / / 2),
$$

and in fact as a spectrum $S / / 2$ is weakly equivalent to a wedge of suspensions of $H \mathbb{F}_{2}$, and a choice of basis for $P_{*}$ determines such a splitting.

We remark that any connective commutative $S$-algebra $E$ for which $0=2 \in$ $\pi_{0}(E)$ admits a morphism of commutative $S$-algebras $u: S / / 2 \rightarrow E$. Using the commutative diagram of $\mathbb{F}_{2}$-algebras

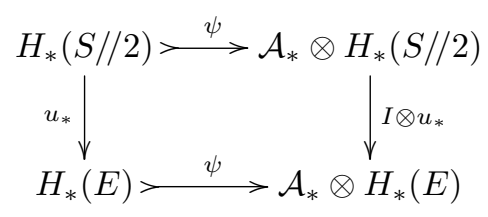

we see that

$\psi\left(u_{*} X_{s}\right)=1 \otimes u_{*} X_{s}+\zeta_{1} \otimes u_{*} X_{s-1}^{2}+\zeta_{2} \otimes u_{*} X_{s-2}^{2^{2}}+\cdots+\zeta_{s} \otimes u_{*} X_{1}^{2^{s}}+\zeta_{s+1} \otimes 1$ 
so the $u_{*} X_{s}$ is a sequence of algebraically independent elements. It follows that there is an isomorphism of $\mathcal{A}_{*}$-comodule algebras

$$
H_{*}(E) \cong \mathcal{A}_{*} \otimes H_{*}(E) /\left(u_{*} X_{s}: s \geqslant 0\right),
$$

so $H_{*}(E)$ is also an extended comodule and $E$ is weakly equivalent to a wedge of suspensions of $H \mathbb{F}_{2}$. This gives a different approach to proving Steinberger's result [7, Theorem III.4.1], which potentially contains more information on the multiplicative structure of the splitting.

Rolf Hoyer has pointed out some explicit formulae for primitives in $H_{*}(S / / 2)$ and thus for families of polynomial generators for $\pi_{*}(S / / 2)$.

\section{$\S 10$. Sample calculations for odd primes}

Now we assume that $p$ is an odd prime and that all spectra are localised at $p$. There are similarities to the 2-primary case, although some of the details are slightly more complicated.

Consider the commutative $S$-algebra $S / / p$ which is the pushout in the diagram of commutative $S$-algebras

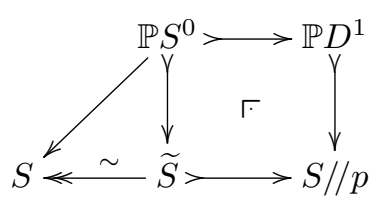

where the notation is similar to that in the case $p=2$. Then $S / / p$ is cofibrant in $\mathscr{C}_{S}$, and there is an isomorphism of commutative $S$-algebras

$$
S / / p \cong \widetilde{S} \wedge_{\mathbb{P} S^{0}} \mathbb{P} D^{1} .
$$

Since the degree $p$ map induces the trivial map in $\bmod p$ ordinary homology, $H_{*}(S / / p)=H_{*}\left(S / / p ; \mathbb{F}_{p}\right)$ can be determined by methods of [3, Theorem 1.7]. The answer is a free graded commutative algebra

$$
H_{*}(S / / p)=\mathbb{F}_{p}\left\langle\mathrm{Q}^{I} x_{1}: I \text { admissible, } \operatorname{exc}(I)>1\right\rangle,
$$

where $x_{1} \in H_{1}(S / / p)$ satisfies $\beta x_{1}=1$.

We define two sequences of elements, beginning with $X_{0}=x_{1}$ and $Y_{0}=1$,

$$
\begin{aligned}
X_{s} & =\mathrm{Q}^{p^{s-1}} X_{s-1}=\mathrm{Q}^{p^{s-1}} \mathrm{Q}^{p^{s-2}} \cdots \mathrm{Q}^{p} \mathrm{Q}^{1} x_{1}, \\
Y_{s} & =\beta \mathrm{Q}^{p^{s-1}} X_{s-1}=\beta \mathrm{Q}^{p^{s-1}} \mathrm{Q}^{p^{s-2}} \cdots \mathrm{Q}^{p} \mathrm{Q}^{1} x_{1} .
\end{aligned}
$$

Notice that the degrees of these elements are $\left|X_{s}\right|=2 p^{s}-1$ and $\left|Y_{s}\right|=2\left(p^{s}-1\right)$. 
We will again use the terminology of strictly allowable introduced in Definition 9.1.

Lemma 10.1. Let $r \geqslant 1$. Then the Dyer-Lashof monomial $\mathrm{Q}^{r} X_{s}$ is strictly allowable only if $r=p^{s}$, while $\mathrm{Q}^{r} Y_{s}$ is never strictly allowable.

Proof. If $\mathrm{Q}^{r} X_{s}=\mathrm{Q}^{r} \mathrm{Q}^{p^{s-1}} X_{s-1}$ is admissible then $r \leqslant p^{s}$, while the required excess condition is $2 r>2 p^{s}-1$.

If $\mathrm{Q}^{r} Y_{s}=\mathrm{Q}^{r} \beta \mathrm{Q}^{p^{s-1}} X_{s-1}$ is admissible then $r<p^{s}$, while the excess condition required for it to be strictly allowable is $2 r>2\left(p^{s}-1\right)$. Clearly these conditions are contradictory.

Proposition 10.2. The left coaction on $X_{s}$ and $Y_{s}$ is given by

$$
\begin{aligned}
\psi X_{s}= & 1 \otimes X_{s}+\bar{\tau}_{0} \otimes Y_{s}+\bar{\tau}_{1} \otimes Y_{s-1}^{p}+\bar{\tau}_{2} \otimes Y_{s-2}^{p^{2}}+\cdots+\bar{\tau}_{s-1} \otimes Y_{1}^{p^{s-1}} \\
& +\bar{\tau}_{s} \otimes 1 \\
\psi Y_{s}= & 1 \otimes Y_{s}+\zeta_{1} \otimes Y_{s-1}^{p}+\zeta_{2} \otimes Y_{s-2}^{p^{2}}+\cdots+\zeta_{s-1} \otimes Y_{1}^{p^{s-1}}+\zeta_{s} \otimes 1 .
\end{aligned}
$$

Proof. Translating the formulae into statements about the right coaction we must prove that the following equations are satisfied for every $s$ :

$$
\begin{aligned}
\widetilde{\psi} X_{s} & =X_{s} \otimes 1+Y_{s} \otimes \tau_{0}+Y_{s-1}^{p} \otimes \tau_{1}+Y_{s-2}^{p^{2}} \otimes \tau_{2}+\cdots+Y_{1}^{p^{s-1}} \otimes \tau_{s-1}+1 \otimes \tau_{s}, \\
\widetilde{\psi} Y_{s} & =Y_{s} \otimes 1+Y_{s-1}^{p} \otimes \xi_{1}+Y_{s-2}^{p^{2}} \otimes \xi_{2}+\cdots+Y_{1}^{p^{s-1}} \otimes \xi_{s-1}+1 \otimes \xi_{s} .
\end{aligned}
$$

Assuming these are true for some $s$, we have

$$
\begin{aligned}
& \widetilde{\psi} X_{s+1}=\widetilde{\psi} \mathrm{Q}^{p^{s}} X_{s}=\mathrm{Q}^{p^{s}}\left(\widetilde{\psi} X_{s}\right)-\beta \mathrm{Q}^{p^{s}}\left(\widetilde{\psi} X_{s}\right) \bar{\tau}_{0} \\
& =\mathrm{Q}^{p^{s}}\left(X_{s} \otimes 1+Y_{s} \otimes \tau_{0}+Y_{s-1}^{p} \otimes \tau_{1}+Y_{s-2}^{p^{2}} \otimes \tau_{2}+\cdots+Y_{1}^{p^{s-1}} \otimes \tau_{s-1}+1 \otimes \tau_{s}\right) \\
& +\beta \mathrm{Q}^{p^{s}}\left(X_{s} \otimes 1+Y_{s} \otimes \tau_{0}+Y_{s-1}^{p} \otimes \tau_{1}+Y_{s-2}^{p^{2}} \otimes \tau_{2}+\cdots+Y_{1}^{p^{s-1}} \otimes \tau_{s-1}+1 \otimes \tau_{s}\right) \tau_{0} \\
& =\left(\mathrm{Q}^{p^{s}} X_{s} \otimes 1+\left(\mathrm{Q}^{p^{s}-1} Y_{s}\right) \otimes \mathrm{Q}^{1} \tau_{0}+\left(\mathrm{Q}^{p^{s-1}-1} Y_{s-1}\right)^{p} \otimes \mathrm{Q}^{p} \tau_{1}\right. \\
& \left.+\left(\mathrm{Q}^{p^{s-2}-1} Y_{s-2}\right)^{p^{2}} \otimes \mathrm{Q}^{p^{2}} \tau_{2}+\cdots+\left(\mathrm{Q}^{p-1} Y_{1}\right)^{p^{s-1}} \otimes \mathrm{Q}^{p^{s-1}} \tau_{s-1}+1 \otimes \mathrm{Q}^{p^{s}} \tau_{s}\right) \\
& +\left(\beta \mathrm{Q}^{p^{s}} X_{s} \otimes 1+\left(\mathrm{Q}^{p^{s}-1} Y_{s}\right) \otimes \beta \mathrm{Q}^{1} \tau_{0}+\left(\mathrm{Q}^{p^{s-1}-1} Y_{s-1}\right)^{p} \otimes \beta \mathrm{Q}^{p} \tau_{1}\right. \\
& \left.+\left(\mathrm{Q}^{p^{s-2}-1} Y_{s-2}\right)^{p^{2}} \otimes \beta \mathrm{Q}^{p^{2}} \tau_{2}+\cdots+\left(\mathrm{Q}^{p-1} Y_{1}\right)^{p^{s-1}} \otimes \beta \mathrm{Q}^{p^{s-1}} \tau_{s-1}+1 \otimes \beta \mathrm{Q}^{p^{s}} \tau_{s}\right) \tau_{0} \\
& =\left(X_{s+1} \otimes 1+Y_{s}^{p} \otimes\left(\tau_{1}-\tau_{0} \xi_{1}\right)+Y_{s-1}^{p^{2}} \otimes\left(\tau_{2}-\tau_{0} \xi_{2}\right)+Y_{s-2}^{p^{3}} \otimes\left(\tau_{3}-\tau_{0} \xi_{3}\right)\right. \\
& \left.+\cdots+Y_{1}^{p^{s}} \otimes\left(\tau_{s}-\tau_{0} \xi_{s}\right)+1 \otimes\left(\tau_{s+1}-\tau_{0} \xi_{s+1}\right)\right) \\
& +\left(Y_{s+1} \otimes 1+Y_{s}^{p} \otimes \xi_{1}+Y_{s-1}^{p^{2}} \otimes \xi_{2}+Y_{s-2}^{p^{3}} \otimes \xi_{3}+\cdots+Y_{1}^{p^{s}} \otimes \xi_{s}+1 \otimes \xi_{s+1}\right) \tau_{0} \\
& =X_{s+1} \otimes 1+Y_{s+1} \otimes \tau_{0}+Y_{s}^{p} \otimes \tau_{1}+Y_{s-1}^{p^{2}} \otimes \tau_{2}+\cdots+Y_{1}^{p^{s}} \otimes \tau_{s}+1 \otimes \tau_{s+1} .
\end{aligned}
$$


A similar calculation shows that

$$
\begin{aligned}
\widetilde{\psi} Y_{s+1}=Y_{s+1} \otimes 1+Y_{s}^{p} \otimes \xi_{1} & +Y_{s-1}^{p^{2}} \otimes \xi_{2} \\
& +\cdots+Y_{2}^{p^{s-1}} \otimes \xi_{s-1}+Y_{1}^{p^{s}} \otimes \xi_{s}+1 \otimes \xi_{s+1} .
\end{aligned}
$$

The result follows by induction.

Let $J=\left(X_{s}, Y_{s+1}: s \geqslant 0\right) \triangleleft H_{*}(S / / p)$ be the ideal generated by the elements $X_{s}, Y_{s}$. As happens for the prime 2 , the following composition of left $\mathcal{A}_{*}$-comodule algebra homomorphisms

$$
H_{*}(S / / p) \underset{\underset{\psi}{\longrightarrow} \mathcal{A}_{*} \otimes H_{*}(S / / p) \underset{\text { quo }}{\longrightarrow} \mathcal{A}_{*}}{\bar{\psi}} \otimes H_{*}(S / / p) / J
$$

is an isomorphism, where the second and third terms are extended left comodules. Here $H_{*}(S / / p) / J$ is a free graded commutative algebra since the generators $X_{s}$ and $Y_{s}$ are amongst the generators of the free graded commutative algebra $H_{*}(S / / p)$. There is a subalgebra $P_{*} \subseteq H_{*}(S / / p)$ which is identified with $\mathbb{F}_{p} \otimes H_{*}(S / / p) / J$ under the isomorphism $\bar{\psi}$, i.e., $\bar{\psi} P_{*}=\mathbb{F}_{p} \otimes H_{*}(S / / p) / J$. A standard argument shows the spectrum $S / / p$ is equivalent to a wedge of suspensions of $H \mathbb{F}_{p}$. As we saw in the 2-primary case, this leads to a proof of Steinberger's result [7, Theorem III.4.1].

\section{Acknowledgements}

I would like to thank the following for their interest and helpful comments: Rolf Hoyer, Nick Kuhn, Tyler Lawson, Peter May, Niko Naumann, Geoffrey Powell, Birgit Richter, John Rognes and Markus Szymik; also the referee deserves special thanks for being so thorough and detecting many errors in formulae.

\section{References}

[1] A. Baker, Husemoller-Witt decompositions and actions of the Steenrod algebra, Proc. Edinburgh Math. Soc. (2) 28 (1985), 271-288. Zbl 0558.55012 MR 0806756

[2] _ Calculating with topological André-Quillen theory, I: Homotopical properties of universal derivations and free commutative $S$-algebras, arXiv:1208.1868 (2012).

[3] $B P$ : Close encounters of the $E_{\infty}$ kind, J. Homotopy Related Structures 9 (2014), 553-578. Zbl 06404317 MR 3258694

[4] T. Bisson and A. Joyal, Nishida relations in bordism and homology, C. R. Math. Rep. Acad. Sci. Canada 17 (1995), 141-146. Zbl 0849.55007 MR 1353741

[5] _ Q Q _ -rings and the homology of the symmetric groups, in Operads: Proceedings of renaissance conferences (Hartford, CT/Luminy, 1995), Contemp. Math. 202, Amer. Math. Soc., 1997, 235-286. Zbl 0877.55011 MR 1436923 
A. BAKER

[6] J. M. Boardman, The eightfold way to $B P$-operations or $E_{*} E$ and all that, in Current trends in algebraic topology, Part 1 (London, Ont., 1981), CMS Conf. Proc. 2, Amer. Math. Soc., 1982, 187-226. Zbl 0563.55002 MR 0686116

[7] R. R. Bruner, J. P. May, J. E. McClure and M. Steinberger, $H_{\infty}$ ring spectra and their applications, Lecture Notes in Math. 1176, Springer, 1986. Zbl 0585.55016 MR 0836132

[8] D. G. Davis and T. Lawson, Commutative ring objects in pro-categories and generalized Moore spectra, Geom. Topol. 18 (2014), 103-140. Zbl 06234228 MR 3158773

[9] E. Dyer and R. K. Lashof, Homology of iterated loop spaces, Amer. J. Math. 84 (1962), 35-88. Zbl 0119.18206 MR 0141112

[10] A. Elmendorf, I. Kříž, M. Mandell and J. P. May, Rings, modules, and algebras in stable homotopy theory, Math. Surveys Monogr. 47, Amer. Math. Soc., 1997. Zbl 0894.55001 MR 1417719

[11] S. O. Kochman, Homology of the classical groups over the Dyer-Lashof algbera, Trans. Amer. Math. Soc. 185 (1973), 83-136. Zbl 0271.57013 MR 0331386

[12] T. Kudo and S. Araki, Topology of $H_{n}$-spaces and $H$-squaring operations, Mem. Fac. Sci. Kyūsyū Univ. Ser. A. 10 (1956), 85-120. Zbl 0074.38502 MR 0087948

[13] N. J. Kuhn, The transfer and James-Hopf invariants. Math. Z. 196 (1987), 391-405. Zbl 0609.55006 MR 0913664

[14] N. J. Kuhn and J. McCarty, The mod 2 homology of infinite loopspaces, Algebr. Geom. Topol. 13 (2013), 687-745. Zbl 06160236 MR 3044591

[15] J. P. May, Homology operations on infinite loop spaces, in Algebraic topology, Proc. Sympos. Pure Math. 22, Amer. Math. Soc., 1971, 171-185. Zbl 0242.55020 MR 0319195

[16] J. Milnor, The Steenrod algebra and its dual, Ann. of Math. (2) 67 (1958), 150-171. Zbl 0080.38003 MR 0099653

[17] G. Nishida, Cohomology operations in iterated loop spaces, Proc. Japan Acad. 44 (1968), 104-109. Zbl 0165.26202 MR 0240811

[18] D. J. Pengelley, The A-algebra structure of Thom spectra: MSO as an example, in Current trends in algebraic topology, Part 1 (London, Ont., 1981), CMS Conf. Proc. 2, Amer. Math. Soc., 1982, 511-513. Zbl 0544.55009 MR 0686134

[19] The mod two homology of MSO and MSU as A comodule algebras, and the cobordism ring, J. London Math. Soc. (2) 25 (1982), 467-472. Zbl 0458.55003 MR 0657503

[20] S. Priddy, Dyer-Lashof operations for the classifying spaces of certain matrix groups, Quart. J. Math. Oxford Ser. (2) 26 (1975), 179-193. Zbl 0311.55013 MR 0375309 